\title{
Laminated connections for structural glass applications under shear loading at different temperatures and strain rates
}

\begin{abstract}
Connections between structural glass components represent one of the main critical aspects of glass engineering. In the last years, a novel typology of adhesive connections has emerged, known as laminated adhesive connections. Two adhesive materials for laminated connections in glass applications are used in this work: the transparent ionomer SentryGlas ${ }^{\circledR}$ (SG) from Kuraray and the Transparent Structural Silicon Adhesive (TSSA) from Dow Corning. Both SG and TSSA show a complex behaviour dependent on strain rate and temperature. This work presents a study that aims (i) to investigate the mechanical behaviour and strength of this connection typology under shear loading and (ii) to quantify the effects of strain rate and temperature on the strength of the connections. This is done by means of a combined experimental, analytical and numerical study on laminated connections made of circular metal connectors bonded to rectangular glass plates. The experimental investigations presented in this work showed that temperature and strain rate variations have important effects on the mechanical response of the connections. Three-dimensional numerical analyses showed a non-uniform stress field with large gradient over the three dimensions. Through analytical studies, prediction models are finally proposed for the shear resistance of TSSA and SG laminated connections. The models are obtained developing an algorithm for multi-dimensional non-linear models with variable standard deviations. A logarithmic law is proposed for the strain rate effects for both TSSA and SG connections. Linear and inverse hyperbolic-tangent-based laws are instead proposed for the TSSA and SG temperature behaviour respectively.
\end{abstract}




\section{Introduction}

The demand for architectural transparency has steadily increased over the last decades. This trend has inevitably promoted the use of glass in buildings and constructions. Due to the fragile nature of this material, connections between structural glass components represent one of the main critical aspects of glass engineering. This is because glass cannot plastically redistribute the stress peaks occurring where forces are transferred between components. Connections for structural glass components can be either bolted or adhesively bonded. Bolted connections make use of metallic bolts placed through drilled holes to transfer forces into the glass components. Mortar or other softer materials are usually placed between glass and metal parts. Adhesive connections are instead realized by joining glass components to metal parts or to other glass components by means of polymer adhesives. In comparison to bolted connections, adhesive connections are characterized by the following advantages: (i) the transfer of forces is distributed over the full bonded area thus avoiding contact stress intensification (ii) the drilling process and the subsequently reduction of the glass strength at the hole edge is avoided (iii) the architectural flushness is enhanced because the metal parts do not go through the glass (iv) thermal bridges and thermal losses are reduced also because the metal part does not go through the entire glass thickness (v) the residual stress field distribution of the tempering is unaltered at the connection and (vi) gas losses occurring in IGU bolted panels are reduced since the glass is not drilled. Because of these aspects, the use of adhesive connections in structural applications has been considered very promising. Indeed, several research projects have focused on adhesive connections for structural glass applications [1]-[11].

In the last years, a novel typology of adhesive connections has emerged, known as laminated adhesive connections. The main characteristic of laminated connections is that they make use of the same production process as applied for laminated glass components. In addition, they exhibit high mechanical performance and are fully transparent. In laminated connections, a solid foil of transparent adhesive material is placed between a metal connector and glass panel. Metal, adhesive and glass are then placed in the vacuum bag and subjected to the standard autoclave process of laminated components. The lamination process is performed by simultaneous application of atmospheric pressure and heat by means of an autoclave. At the end of the lamination process the result is a glass component where the metal part is fully bonded to the glass plate by means of the laminated transparent adhesive.

Two adhesive materials for laminated connections in glass applications are used in this work: the transparent ionomer SentryGlas ${ }^{\circledR}$ (SG) from Kuraray and the Transparent Structural Silicon Adhesive (TSSA) from Dow Corning. More detailed information on these materials and existing literature on laminated connections are given in the following sections. Table 1 collects a summary of the basic material properties provided by standards and material producers for SG, TSSA and other material properties used in this work. Further material properties used in this work are taken for the experimental investigation performed in [12]. Laminated connections have been used in several projects worldwide. Applications of SG laminated connections can be found [13]-[18] and TSSA applications in [19]. 
Table 1: Materials properties provided by standards and material producers.

\begin{tabular}{|c|c|c|c|c|c|c|}
\hline Property & Density & $\alpha_{T}$ & $E$ & $v$ & $\sigma_{\max }$ & $\varepsilon_{\max }$ \\
\hline Unit & $\mathrm{g} / \mathrm{cm}^{3}$ & $10^{-5} /{ }^{\circ} \mathrm{C}$ & $M P a$ & - & $M P a$ & $\%$ \\
\hline $\mathrm{SG}^{a}$ & 0.95 & $15-10$ & $692-0.5$ & $0.5-0.4$ & 34.5 & 400 \\
\hline $\operatorname{TSSA}^{b}$ & $\mathrm{n} / \mathrm{a}$ & $\mathrm{n} / \mathrm{a}$ & $9.0-4.5$ & $\mathrm{n} / \mathrm{a}$ & 8.5 & 250 \\
\hline Glass $^{c}$ & 2.50 & 9 & 70000 & 0.23 & $45^{e}$ & 0.06 \\
\hline Stainless steel $1.4404^{d}$ & 7.85 & 16 & 200000 & 0.3 & $530^{f}$ & 40 \\
\hline
\end{tabular}

a) $[20][21]^{1}$ b) [22] c) [23]-[25] d) [26], [27] e) characteristic equi-biaxial bending stress at $2 \mathrm{MPa} / \mathrm{s} \mathrm{f}$ ) ultimate stress

\subsection{Ionomer SentryGlas® (SG)}

SentryGlas ${ }^{\circledR}(\mathrm{SG})$ is a thermoplastic transparent ionomer polymer used in laminated glass applications as interlayer. The glass transition temperature of $\mathrm{SG}$ is at $50-55^{\circ} \mathrm{C}[28][29]^{2}$. Compared to other interlayers such as PVB and EVA, SG is characterized by higher stiffness, enhanced durability and higher mechanical resistance. From a chemical point of view, ionomers are polymeric materials in which the main repetitive sequence of monomers is characterized by additional ionic groups. Ionomers belong to the category of polyelectrolyte. More specifically, ionomers are often defined as polyelectrolytes with ionic groups not exceeding $20 \mathrm{~mol} \%$ [30]. Other authors, instead, more generally defines ionomers as polymeric materials with mechanical performance influenced by the ionic group interactions and the subsequent formation of ionic aggregates [31]. It is indeed the attraction between ionic groups and the subsequent cross-links between polymeric chains that enhances the physical properties and the mechanical response of the polymer [32]. The SG is currently produced in foil thickness of $0.72,0.89$ and $1.52 \mathrm{~mm}$. SG foils are rather rigid at room temperature and usually require the use of sharp tools to cut the foil to the desired size and geometry. In the production of laminated glass connections, glass, SG foil and metal parts are placed in a vacuum bag and subjected to an autoclave process ${ }^{3}$. The lamination process consists in a single cycle of simultaneous application of heat and pressure. Typically, a temperature of $135^{\circ} \mathrm{C}$ and a pressure of 12 bar are applied for a minimum plateau time of 60 minutes $^{4}$. Subsequently, to achieve a good lamination quality, the cooling phase should be performed with a minimum rate of $2-3^{\circ} \mathrm{C} / \mathrm{min}$. At the end of the autoclave process, the SG material is fully transparent ${ }^{5}$.

Several authors have investigated the mechanical response of SG-laminated components [33]-[44] and the SG-bulk material [12], [45]-[51]. Conversely, studies on the resistance of SG laminated connections are rather limited. Exploratory tests on SG laminated connections bonded to the glass surfaces are performed by Peters in [52]. In [52], a rectangular metal connector is bonded to the surface of a laminated glass panel. Tests are then performed clamping the glass panel and applying tensile force to the metal connection. Tests are performed at room temperature. In the work performed by Belis et al. [53]-[55] a broad screening of adhesive connections is performed via a large experimental campaign to select promising adhesives for glass applications. Tests are performed on aluminium-glass single lap joints at reference condition and after exposition to artificial aging protocols (4 and 12 weeks exposition to $90 \%$ R.H and $50^{\circ} \mathrm{C}$ ). Tests were performed at room temperature. Based on the experimental observation, SG connections have been indicated, among

\footnotetext{
${ }^{1}$ It should be noticed that these values are time and temperature dependent.

${ }^{2}$ This is higher than other common interlayer polymers used in laminated components, such as standard PVB with a typical glass transition temperature around $15-20^{\circ} \mathrm{C}$.

${ }^{3}$ As an alternative to the autoclave-vacuum process, silicon bag are also used. In these cases, the components are placed inside a vacuumized silicon bag that is then placed inside a oven. This process is often commercially indicated as Tema.

${ }^{4}$ Material producer suggests that optimum values of temperature and pressure depend on the several factors and vary among different glass manufactures (e.g. autoclave size, panel size, factory, etc...). Therefore, the values mentioned in this manuscript must be considered to be only indicatives. For more details the reader should refer to the material producer or certified glass manufactures.

${ }^{5}$ However, it should be noticed that before lamination the SG foils appear not fully transparent because of the micro-channels intentionally realized on the SG surfaces. These micro-channels reduce the risk of air-bubble inclusion since the air can flow out of the component during the lamination.
} 
others, as a promising candidate for adhesive connections. In the work of Watson and Overend [56], an extensive work is performed testing single lap connections with different adhesive and interlayers, among which SG. The results showed that SG connections exhibit one of the largest load carrying capacity, often limited by glass failure. Limited results are available in literature at different temperatures [17], [46], [57]-[63]. Results showed that the temperature has a dominant effect on the mechanical response of the connection, with a severe reduction of the maximum load-carrying capacity at high temperature. Results also showed that, at room temperature, the maximum capacity of the connection is limited by the plastification of the metal insert or glass breakage.

\subsection{Transparent Structural Silicon Adhesive (TSSA)}

The TSSA material is a Transparent Structural Silicon Adhesive elastomer, produced by Dow Corning, that has been recently commercialized for laminated connections in structural glass applications. Compared to standard silicone adhesives used in glass applications, TSSA exhibits higher stiffness and strength, which makes it suitable for structural applications. It should be noticed that, conversely to SG, TSSA is exclusively intended to realize metal-to-glass adhesive laminated connections glued to the glass surface. TSSA is not intended for laminated glass components in general. TSSA is a one-component addition-cured silicon with no by-products, characterized by nanosilica and cross-linked polymers. The curing chemical reaction occurs between $\mathrm{Si}-\mathrm{H}$ containing polymers and Si-Vinyl containing polymer in the presence of platinum with addition cure hydrosilylation $(\mathrm{Si}-\mathrm{H}+\mathrm{Si}-\mathrm{Vinyl}=\mathrm{Si}-\mathrm{CH} 2-\mathrm{CH} 2-\mathrm{Si})^{6}$. TSSA is characterized ${ }^{7}$ by an amorphous structure since crystallization that starts below $-55^{\circ} \mathrm{C}$. The glass transition temperature of the polymers is around $-120^{\circ} \mathrm{C}^{6}$. The stiffness of TSSA is therefore stable against temperature variation. The curing process is activated by heat and it occurs rather rapidly. Rheometry tests showed that $90 \%$ of the mechanical response is achieved after 15 minutes at $130^{\circ} \mathrm{C}$ [64]. However, a minimum of 1 hour at $130^{\circ} \mathrm{C}$ is recommended to achieve uniform curing. Given its aforementioned characteristics, it follows that the use of TSSA adhesive in laminated glass application is quite convenient. Indeed, the autoclave pressure ensures good contact between the adhesive and the adherend surfaces and the heat provided in the lamination cycle activates the addition-cured curing. TSSA is produced in foils of $1 \mathrm{~mm}$ thickness foil to be stored at low temperature. The specimens preparation is performed by (i) application of the TSSA to the metal connector (ii) cut of the excessive material out of the metal connector (iii) application to the glass surface and (iv) application of pre-pressure. The producer suggest to apply a pre-pressure in the range of $0.15-1.3 \mathrm{MPa}$ [19]. This helps to prevent air inclusions and to ensure good contact between the TSSA and the adherend surfaces. Both glass and metal connector must be cleaned by means of cleaning agent (e.g. isopropyl alcohol) and silane primer.

In the work of Watson \& Overend [56] experimental investigations on TSSA are performed. More specifically, TSSA single lap joints and T-peel specimens are tested at room temperature. The experimental results indicate large potentiality of the TSSA connections because its mechanical response is characterised by an efficient compromise between flexibility and load carrying capacity. In the work of Sitte et al. [64], [65] circular TSSA connections are tested under tensile and shear load. Tests are mainly performed at room temperature and constant displacement rate. Some exploratory investigations are also performed at different temperature. The preliminary results indicate that the temperature has significant effects on the resistance of TSSA connections. TSSA bulk material is also investigated at room temperature and constant displacement rate. In the work of Wolf et al. [66] the aging of TSSA connections is extensively investigated. Connections are exposed to both outdoor exposition and accelerated aging protocols. Accelerated aging protocols involved U.V. radiation, water immersion and high temperature cycling. The results show that the TSSA preforms well against

\footnotetext{
${ }^{6}$ P.V. Dow Corning Europe, Personal Communication, July $22^{\text {th }}, 2015$.

${ }^{7}$ Within the common range of temperatures for structural applications, e.g. $-20^{\circ} \mathrm{C}+80^{\circ} \mathrm{C}$ according to ETAG 002 [68]
} 
aging exposition, as expected for silicon material because of its high energy SI-O bonds [67]. In some cases, it is even observed that aging exposition induces enhancement of the mechanical resistance of TSSA connections. This could indicate that either some further curing is occurring over time or that the aging effect is quantitatively more limited than the statistical dispersion of the results.

\subsection{Objectives}

The preliminary investigations available in literature indicates that SG and TSSA shows a complex behaviour dependent of strain rate and temperature. However, despite their use in several project, limited information is available on their mechanical resistance and on the effects of strain rate and temperature variation. The aims of this work are therefore to (i) to increase the understanding of the mechanical behaviour and strength of this connection typology under shear loading and (ii) to quantify the effects and strain rate and temperature on the mechanical strength of the connections. This is done by means of a combined experimental, analytical and numerical study on laminated connections made of circular metal connectors bonded to rectangular glass plates.

More in detail, in the following sections from 2 to 4, TSSA and SG laminated connections are investigated through an extensive experimental campaign. The specimens are loaded with pure shear loading up to failure at different temperatures and displacement rates. Then, in section 5, threedimensional numerical analyses of laminated connections are performed by means of finite element method. This is done to accurately compute the stress distribution in the connection, with particular attention to the computation of stress peaks and the stress field distribution in the adhesives. A parametrical study is also performed to evaluate the effects of the adhesive stiffness on the adhesive stress distribution. In section 6, a simplified two-dimensional variational approach is used to obtain an analytical expression of the stress profile in the adhesive. This is done to understand the physics behind the non-linear stress distribution and to analytically determine how different parameters affect the stress distribution in the adhesive. Finally, in section 7, failure prediction models are proposed for the shear resistance of SG and TSSA laminated connections. The models provide the connections resistance with analytical equation expressed as function of the applied strain rate and the temperature. 


\section{Experimental campaign}

\subsection{Specimens and setup}

The laminated connections tested in this work are obtained bonding metal connectors to glass plates via laminated transparent adhesive polymers. The adhesive materials are TSSA silicon and SG ionomer. The TSSA nominal thickness is $1 \mathrm{~mm}$. The SG nominal thickness is $1.5 \mathrm{~mm}$.

Solid metal connectors are machined out of a solid metal bar of $50 \mathrm{~mm}$ diameter, with a height of $20 \mathrm{~mm}$ and tolerance h9 [ISO 286] (see Figure 1). The bonded surface is machined with roughness of 8 micron. A $10 \mathrm{~mm}$ blind threated hole is machined along the connector axis with a depth of $15 \mathrm{~mm}$. Two main reasons motivated the choice of circular shape for the metal connector. Firstly, a circular shaped connector avoids stress intensification at the corners that occurs, for instance, in rectangular connectors. Secondly, with circular connectors, the orientation and alignment of the metal connector to the glass edge is not critical. This facilitates the production process and the testing of laminated connections. Metal connectors are made of stainless steel $316 \mathrm{~L}^{8}$, a commonly used material for connections in facades and structural glass applications.
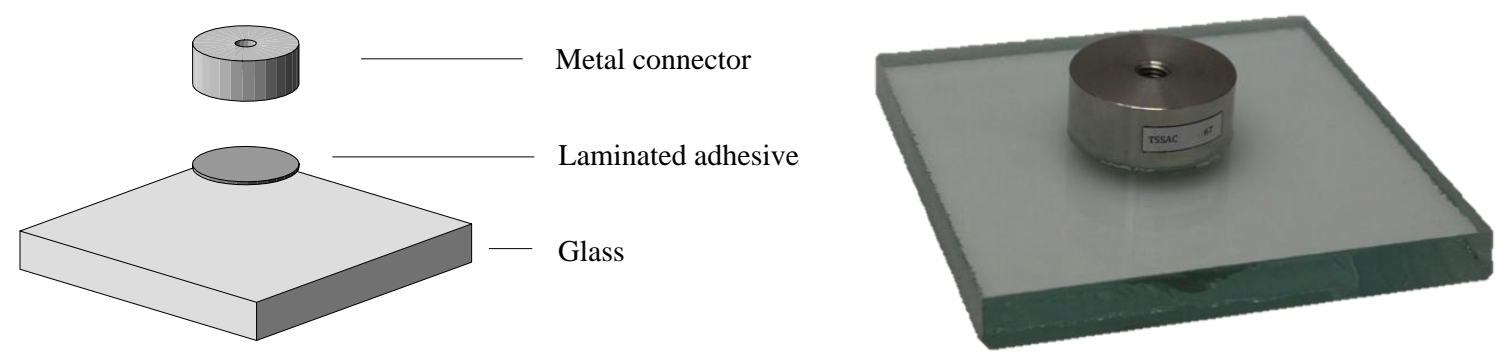

Figure 1: Scheme and photo of the specimen used in the shear tests

Annealed glass plates of $150 \mathrm{~mm} \times 150 \mathrm{~mm}$ and thickness of $15 \mathrm{~mm}$ are used for TSSA connections. Tempered glass plates of $300 \mathrm{~mm} \times 150 \mathrm{~mm}$ and thickness of $19 \mathrm{~mm}$ are used for SG connections. Analytical calculation and exploratory investigations showed that the use of annealed glass for SG connections would induce glass failure before the adhesive. This would not allow to obtain information on the adhesive mechanical properties. A $19 \mathrm{~mm}$ tempered glass is therefore chosen to reduce as much as possible the risk of glass failure. The longer dimension of $300 \mathrm{~mm}$ is the minimum size that can be tempered in a standard tempering line'.

\footnotetext{
${ }^{8}$ The $316 \mathrm{~L}$ alloy is an austenitic stainless steel characterized by a better corrosion resistance than the common 304 . The suffix "L" stands for low carbon (i.e. $<0.03 \%$ ) and indicates better weldability performances. This particular stainless steel has indeed reduced risk of intergranular corrosion related to carbides precipitations at the grains boundaries after welding.

${ }^{9}$ The distances between rollers of tempering oven is about $28 \mathrm{~cm}$.
} 

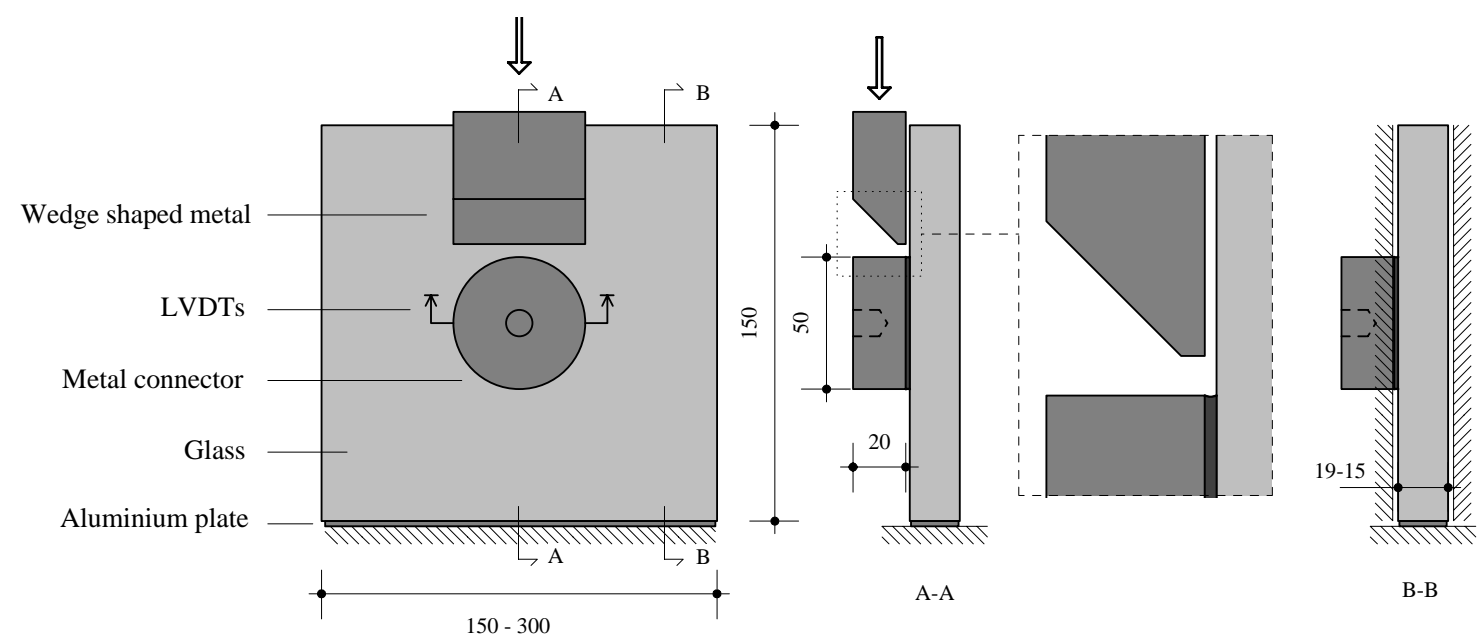

B-B

Figure 2: Specimens geometry, load application and boundary condition for shear tests

Tests are performed with a $50 \mathrm{kN}$ Universal Testing machine with a $50 \mathrm{kN}$ load cell with accuracy class 0.02. The machine is equipped with a climatic chamber of range $-30^{\circ} \mathrm{C}+80^{\circ} \mathrm{C}$ with a resolution of $0.1^{\circ} \mathrm{C}$. A custom made steel setup is fabricated and installed in the machine for the correct introduction of load and to ensure high setup rigidity (see Figure 3). The shear load is applied to the specimen by means of a wedge shaped metal element, attached to the upper part of the machine. The wedge edge is aligned to the interface between the metal and the adhesive (see Figure 2). This is done to apply a pure shear load to the specimen and to reduce to the minimum the eccentricity between load and glass surface. It should be noticed that the minimum value of eccentricity is equal to the adhesive thickness. Firstly, the specimen is inserted in the metal setup (see Figure 3 (a)). Then the specimen is clamped with rigid plate pushed against the glass plate by adjustable bolts threaded in the setup. This system allows to clamp different dimensions and thicknesses of glass plate. Thin aluminium plates are placed between the glass and the steel setup. The back plate of the metal setup has circular hole at the level of the connection to allow visual inspection and video recording of the adhesive through the glass during the test (Figure 3 (c)). The metal setup is then bolted to a thick metal base and "framed" to the base by trapezoidal shaped solid steel stiffeners (Figure 3 (b)).

(a)

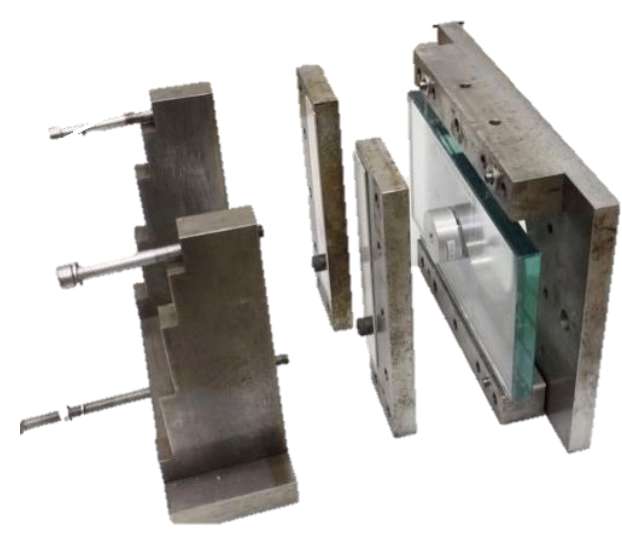

(b)

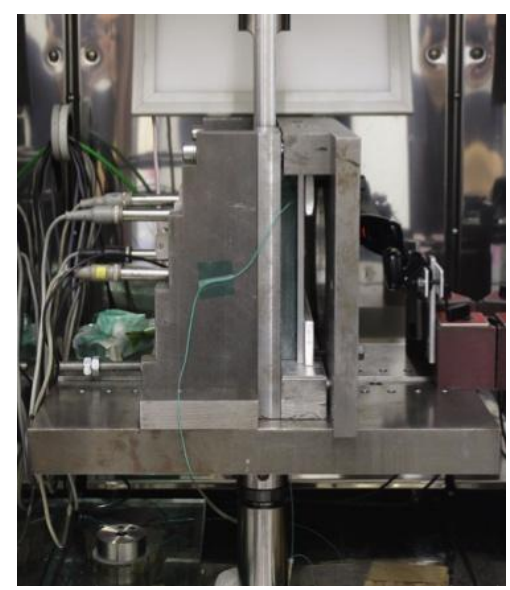

(c)

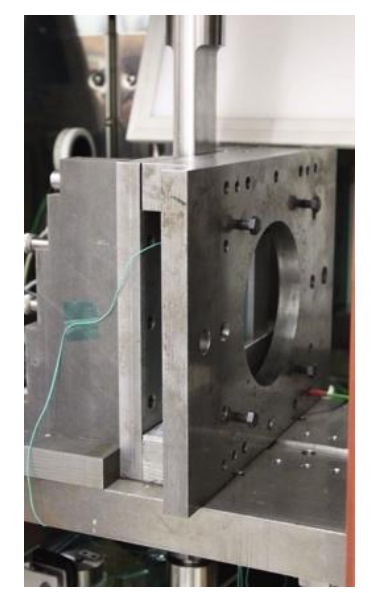

Figure 3: Photos of test setup: (a) test setup assembly (b) test setup inside the climatic chamber (c) back view of the test setup in the climatic chamber

The applied load, machine crosshead displacement and relative displacement between glass and metal connector are measured during the test. Displacements are measured by two inductive LVDTs of \pm $5 \mathrm{~mm}$, directly attached to the metal connector (see Figure 2). Data are acquired with a frequency of 
$100 \mathrm{~Hz}$. A video camera is installed inside the climatic chamber. The camera is placed behind the glass plate (see Figure 4) for video recording of the adhesive during the test.

Exploratory tests has shown that the cooling/heating rates of the specimen and the climatic chamber show non-negligible differences. An additional system for accurate temperature measurements is therefore required. A total number of five thermocouples were used during each test (see green wires in the photos of Figure 3 (b) and Figure 3 (c)). The first four thermocouples are use to measure (i) the temperature of the glass close to the adhesive (ii) the temperature of the metal connector close to the adhesive (iii) the temperature of the metal setup and (iv) the temperature of the air in the climatic chamber. The fifth thermocouple is laminated within the adhesive in a reference specimen that is kept in the climatic chamber during each test. The use of these thermocouples permit to control and ensure uniform temperature everywhere during the test. Indeed, each test is started only when each thermocouple has reached the targeted temperature. 


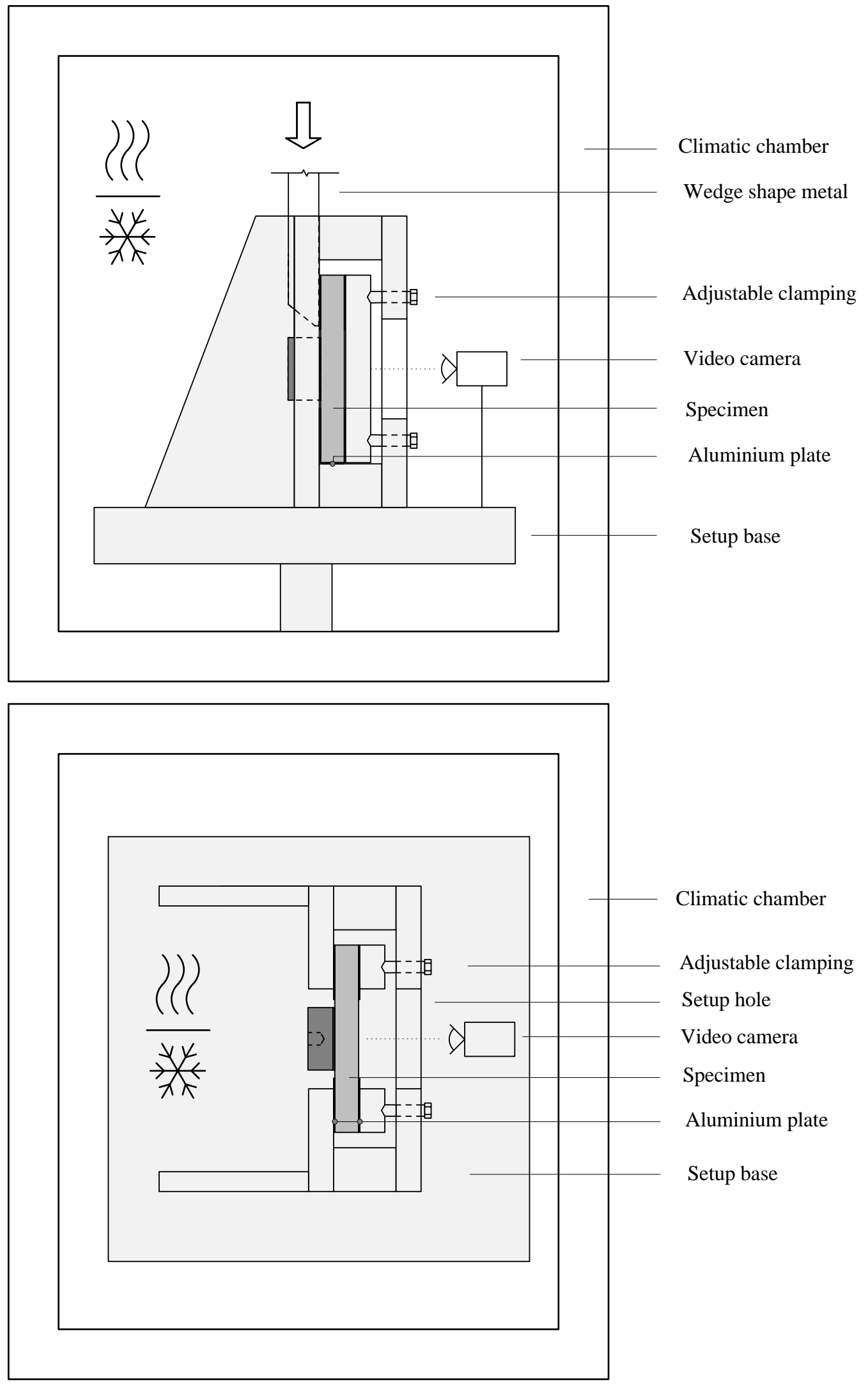

Figure 4: Scheme of shear test setup: frontal and plan view 


\subsection{Test configuration}

According to the guideline ETAG 002 [68], $-20^{\circ} \mathrm{C}^{10}$ and $80^{\circ} \mathrm{C}$ are considered as temperature limits for practical purpose in civil engineering, while $23^{\circ} \mathrm{C}$ is considered the reference value. According to this indication, TSSA silicon is tested at $-20^{\circ} \mathrm{C}, 23^{\circ} \mathrm{C}$ and $80^{\circ} \mathrm{C}$. An intermediate temperature of $50^{\circ} \mathrm{C}$ is also tested. SG instead is tested at 7 different temperatures within this range: $-20^{\circ} \mathrm{C}, 0^{\circ} \mathrm{C}, 23^{\circ} \mathrm{C}, 40^{\circ} \mathrm{C}$, $50^{\circ} \mathrm{C}, 60^{\circ} \mathrm{C}$ and $80^{\circ} \mathrm{C}$. SG is tested at more temperatures due to its high temperature sensitivity.

Table 2: Test configurations for TSSA laminated connections under shear load

\begin{tabular}{ccccccccc}
\hline Material & {$[\mathrm{mm} / \mathrm{min}]$} & $-20^{\circ} \mathrm{C}$ & $0^{\circ} \mathrm{C}$ & $23^{\circ} \mathrm{C}$ & $40^{\circ} \mathrm{C}$ & $50^{\circ} \mathrm{C}$ & $60^{\circ} \mathrm{C}$ & $80^{\circ} \mathrm{C}$ \\
\hline \multirow{3}{*}{ TSSA } & 0.1 & - & - & 1 & - & 1 & - & 1 \\
& 1 & 3 & - & 5 & - & 3 & - & 3 \\
& 10 & - & - & 1 & - & 1 & - & 1 \\
\multirow{2}{*}{ SG } & 0.1 & - & - & 1 & - & 1 & - & 1 \\
& 1 & 3 & 3 & 5 & 3 & 3 & 3 & 3 \\
& 10 & - & - & 1 & - & 1 & - & 1 \\
\hline
\end{tabular}

Tests are performed in displacement control. Both materials are tested at three different crosshead machine displacement rates: $0.1 \mathrm{~mm} / \mathrm{min}, 1 \mathrm{~mm} / \mathrm{min}$ and $10 \mathrm{~mm} / \mathrm{min}$. The maximum and minimum displacement rates are defined by practical limitation. Tests at different displacements rate are performed at $23^{\circ} \mathrm{C}, 50^{\circ} \mathrm{C}$ and $80^{\circ} \mathrm{C}$. Tests at $1 \mathrm{~mm} / \mathrm{min}$ are repeated at the same configuration to evaluate statistic spread of results (at least five tests at $23^{\circ} \mathrm{C}$ and at least three tests at the other temperatures). The investigated configurations are summarized in Table 2 for TSSA and SG. A total number of 49 tests are presented in this work

\subsection{Experimental results}

\subsubsection{TSSA connections under shear load}

Figure 5 shows the results of shear tests on TSSA laminated connections at different temperatures. The mechanical response of TSSA connections under shear load is observed to be mainly linear from the beginning of the test up to failure. This is in agreement with [64], [69]. Large deformations are exhibited during the tests with ultimate displacement exceeding $3 \mathrm{~mm}$. Temperature variations do not affect the global behaviour of the connections. Indeed, the curves at $-20^{\circ} \mathrm{C}, 23^{\circ} \mathrm{C}, 50^{\circ} \mathrm{C}$ and $80^{\circ} \mathrm{C}$ are overlapping. This is in line with the preliminary observation of [64]. However, the variation of temperature has effects on the resistance of the connection. More specifically, the maximum load increases when the temperature decreases. The range of values goes from $8 \mathrm{kN}$ to $13 \mathrm{kN}$. From the video recording it is observed that, conversely to what happens in tensile test (see [70]), the adhesive does not show a clear whitening effect. Only few small dots are visible before failure. The failure mode is always fully cohesive. After the test, the surfaces of the adhesive look slightly different for different temperatures (see Figure 6). At high temperature the adhesive surface is rougher. Glass failure is never occurring. The full set of results are collected and plotted in Appendix A.

\footnotetext{
${ }^{10}$ According to ETAG 002 low temperature limit could be extended down to $-40^{\circ} \mathrm{C}$ for European Nordic countries if required
} 
(a)

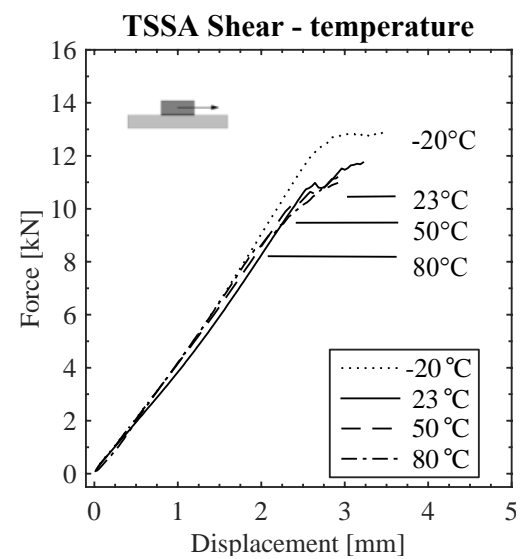

(b)

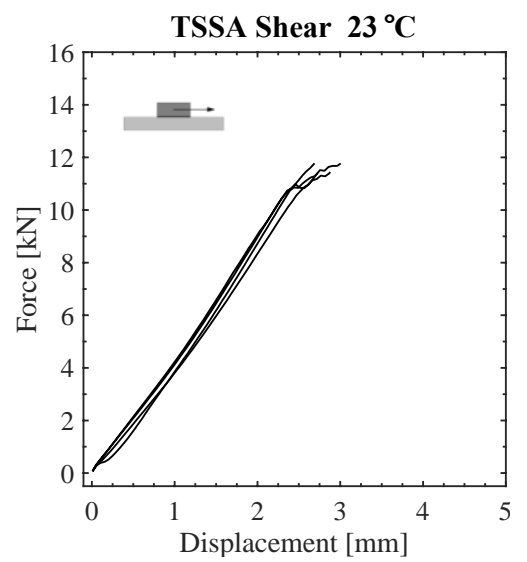

Figure 5: TSSA laminated connections under shear force at $1 \mathrm{~mm} / \mathrm{min}$ (a) Test results at $-20^{\circ} \mathrm{C}, 23^{\circ} \mathrm{C}, 50^{\circ} \mathrm{C}$ and $80^{\circ} \mathrm{C}$ (b) Test results of five specimens at $23^{\circ} \mathrm{C}$

(a)

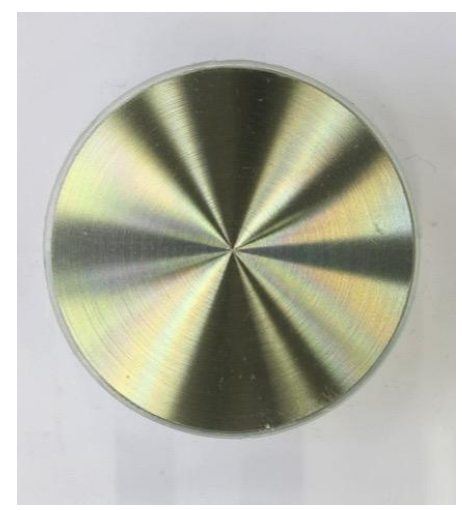

(b)

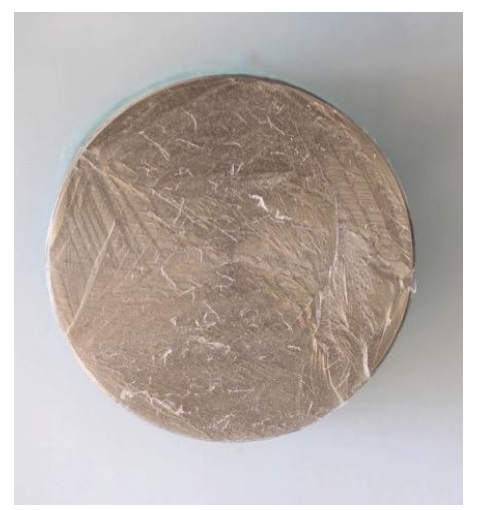

(c)

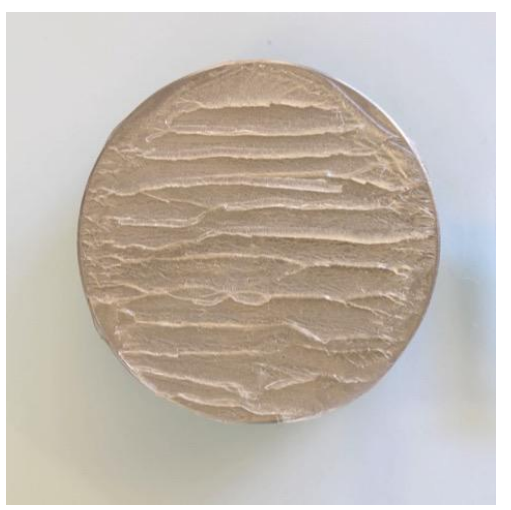

Figure 6: TSSA laminated connections before and after shear tests. Load is applied along vertical direction. (a) Specimens before testing (b) tested specimen at $23^{\circ} \mathrm{C}$ (c) tested specimen at $80^{\circ} \mathrm{C}$

\subsubsection{SG ionomer under shear load}

Figure 7 shows the tests results on SG ionomer laminated connections at different temperatures. The mechanical response of SG connections appears to be more sensitive to temperature changes than TSSA one. At first, the mechanical response is linear at any temperature. The slope of the linear part is temperature dependent, with very rigid response at low temperature and soft response at high temperature. After the linear part, the SG always exhibits yielding behaviour. This is visible by the deviation of the curve from the linear behaviour. The behaviour after the yielding depends on the temperature.

At high temperatures (i.e. larger than $23^{\circ} \mathrm{C}$ ) the connections show large deformation up to cohesive failure of the adhesive (see Figure 9). Glass failure is not occurring. At room and low temperatures instead, the yielding phase is followed by failure of the adherend, i.e. glass breakage. After breakage, the adhesive remains fully attached to the metal connector. Figure 8 shows a representative SG connection after being tested at room and low temperatures. In some cases, the glass fracture propagates over the whole panel in an explosive manner, as usually expected for fully tempered glass (Figure 10). In other cases, the crack in the glass is stable and does not fully propagate over the panel (see Figure 8 (a)). In these cases, the crack starts at the bonded surface of the glass and then propagates downwards through the thicknesses up to a certain extent. Then, it deviates and goes back to the bonded surface of the glass. The full set of results are collected and plotted in Appendix A. 
(a)

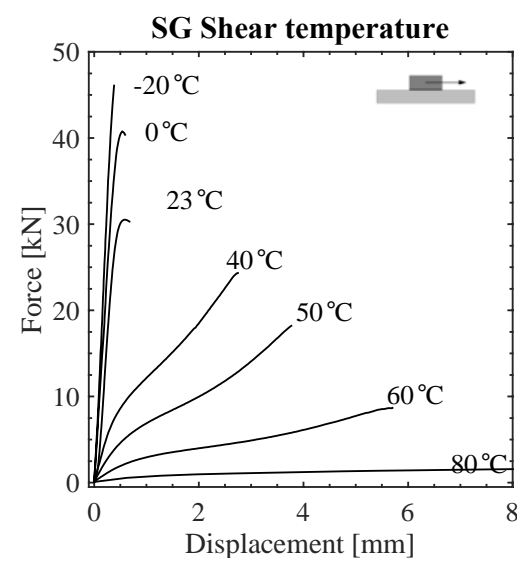

(b)

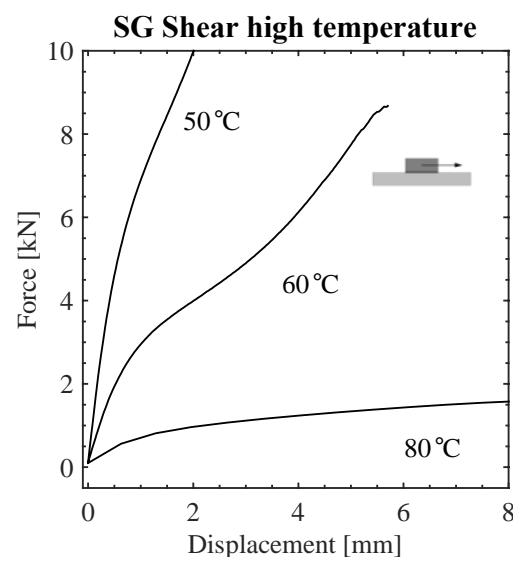

Figure 7: SG laminated connections under shear force at $1 \mathrm{~mm} / \mathrm{min}$ (a) Test results at $-20^{\circ} \mathrm{C}, 0^{\circ} \mathrm{C}, 23^{\circ} \mathrm{C}, 40^{\circ} \mathrm{C}, 50^{\circ} \mathrm{C}, 60^{\circ} \mathrm{C}$ and $80^{\circ} \mathrm{C}$ (b) Zoom at high temperatures.

(a)

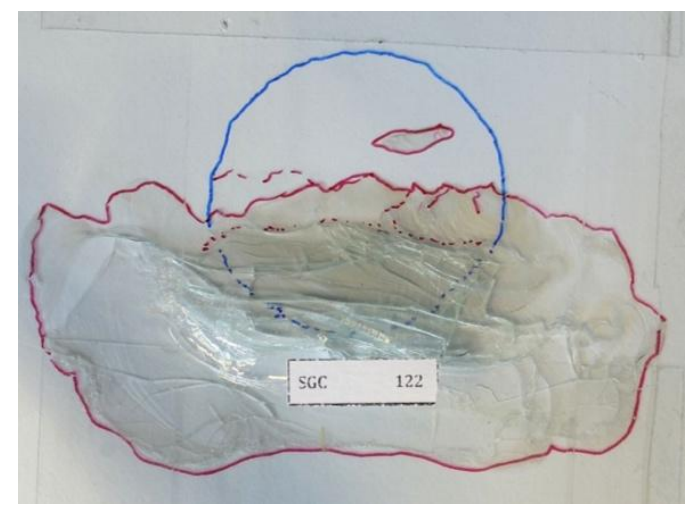

(b)

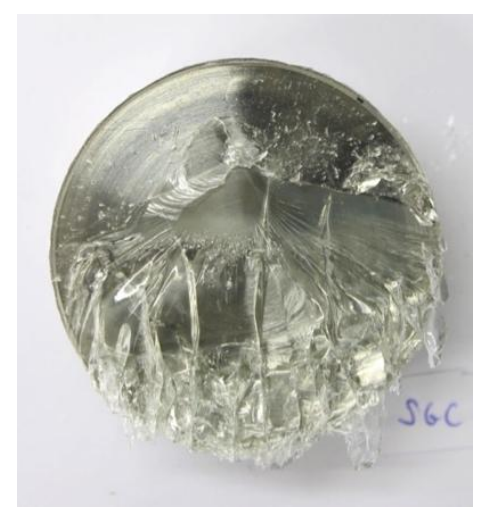

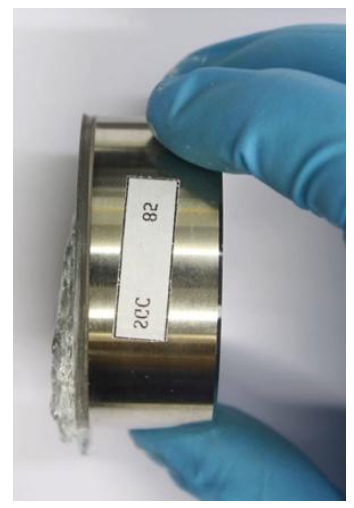

Figure 8: SG laminated connections at $23^{\circ} \mathrm{C}$ under shear force. Load is applied along vertical direction (a) Tested specimens: glass plate without crack propagation in the whole glass panel (b) Tested specimen: metal connector (c) Tested specimen: metal connector, lateral view

(a)

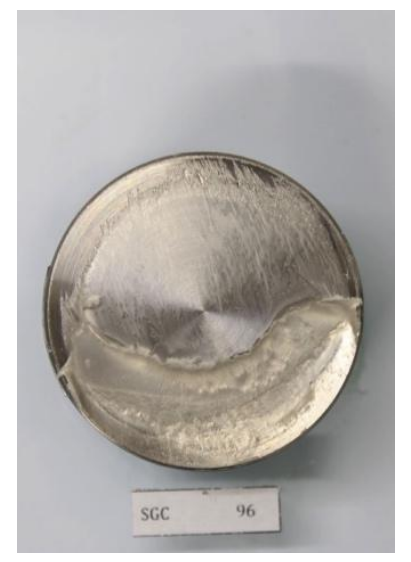

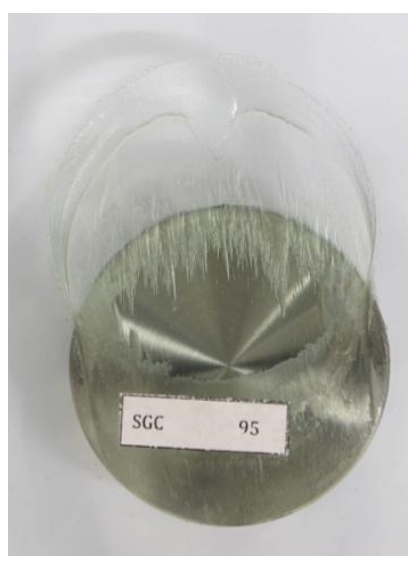

(b)

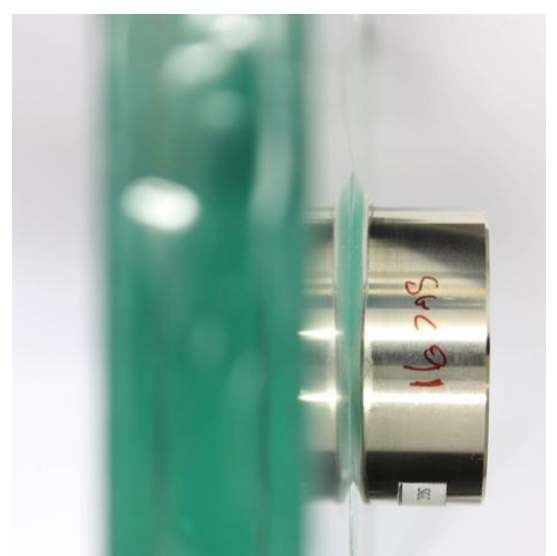

Figure 9: SG laminated connections at $80^{\circ} \mathrm{C}$ under shear force. Load is applied along vertical direction. (a) Plan views of tested specimens (b) Lateral view of tested specimen 


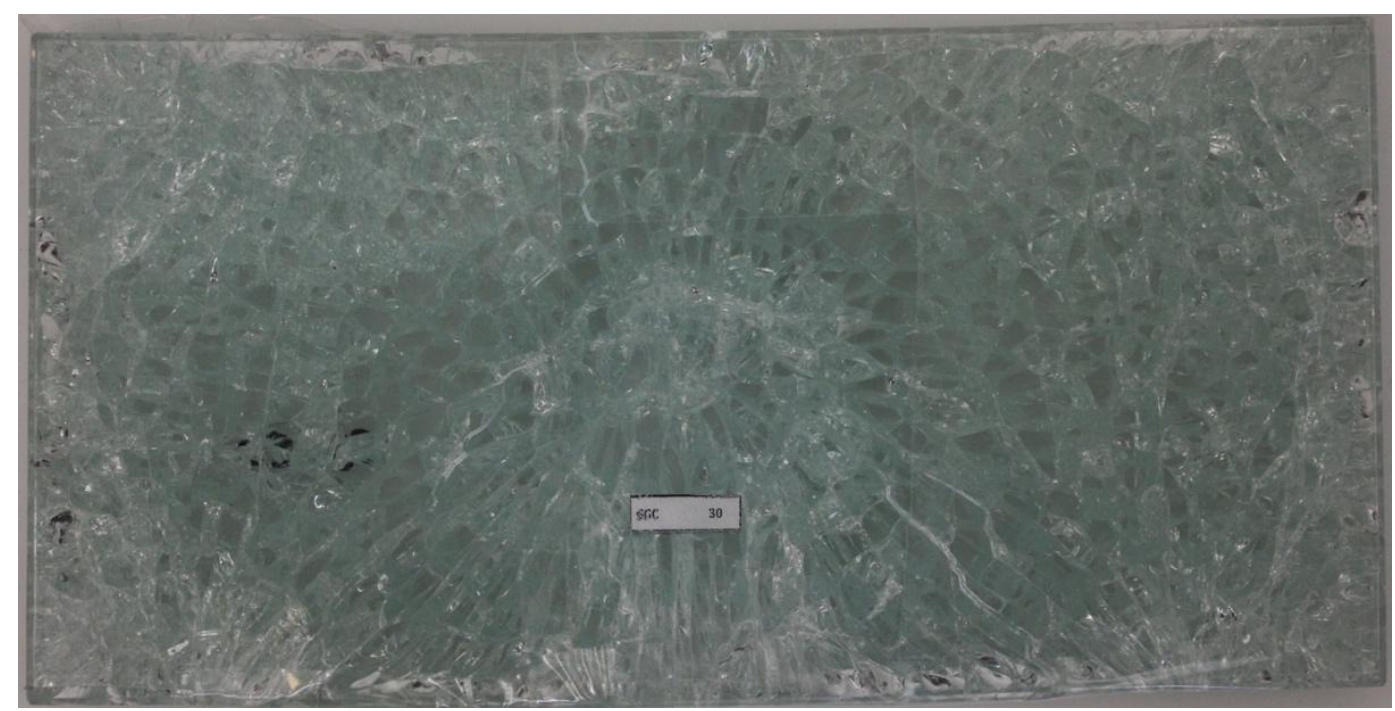

Figure 10: Photo of fully broken glass specimen of a SG laminated connection after shear test at room and low temperatures

\subsection{Discussion of experimental results}

Figure 11 shows shear resistance of TSSA connections plotted versus temperature and strain rate. The average values of resistance, displacement rate, strain rate and temperature are collected by Table 3 . Table 4 provides than the standard deviation and the coefficient of variation for different temperatures. Preliminary investigations performed in [64] indicate that the TSSA resistance changes at $80^{\circ} \mathrm{C}$, which is confirmed by the experimental campaign presented in this work. More specifically, the resistance appear to follow a linear law with the temperature, increases up to $24 \%$ at $-20^{\circ} \mathrm{C}$ and decrease up to $22 \%$ at $80^{\circ} \mathrm{C}$ compared to room temperature tests. The results indicate that the resistance is also strain rate dependent and appears to follows a logarithmic law. The standard deviation is variable with the temperature with coefficients of variation between 5\% to 7\%. The scatter of the response appears to be moderate and slightly increasing with temperature (see Table 4).

(a)

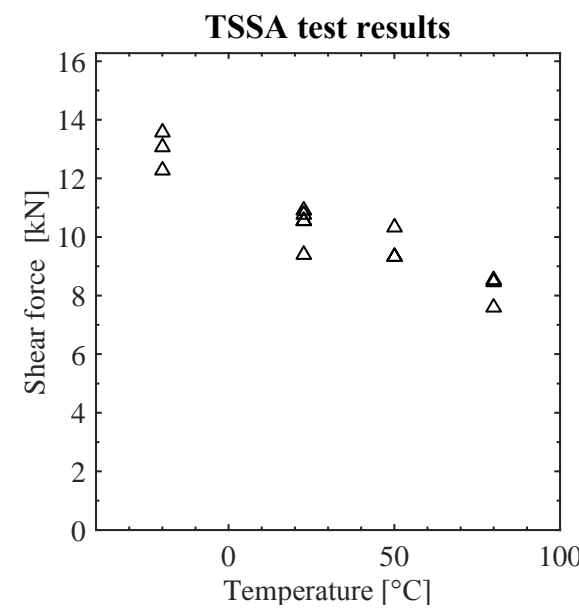

(b)

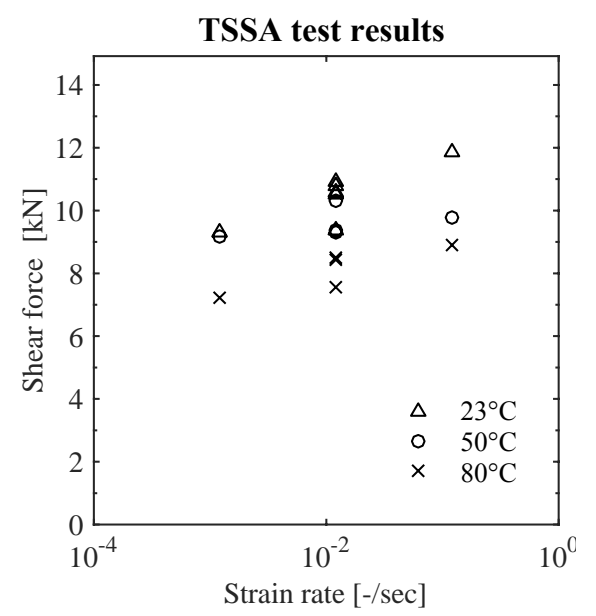

Figure 11: TSSA test results plotted versus (a) temperature (b) strain rate 
Table 3: Test results of TSSA laminated connections - mean values

\begin{tabular}{cccc}
\hline $\mathrm{T}$ & $\dot{d}$ & $\dot{\gamma}$ & $F_{V}$ \\
{$\left[{ }^{\circ} \mathrm{C}\right]$} & {$[\mathrm{mm} / \mathrm{min}]$} & {$[-/ \mathrm{sec}]$} & {$[\mathrm{kN}]$} \\
\hline-20 & 1 & $1.2 \mathrm{E}-02$ & 12.96 \\
23 & 0.1 & $1.2 \mathrm{E}-03$ & 9.32 \\
23 & 1 & $1.2 \mathrm{E}-02$ & 10.42 \\
23 & 10 & $1.2 \mathrm{E}-01$ & 11.86 \\
50 & 0.1 & $1.2 \mathrm{E}-03$ & 9.15 \\
50 & 1 & $1.2 \mathrm{E}-02$ & 9.65 \\
50 & 10 & $1.2 \mathrm{E}-01$ & 9.79 \\
80 & 0.1 & $1.2 \mathrm{E}-03$ & 7.22 \\
80 & 1 & $1.2 \mathrm{E}-02$ & 8.17 \\
80 & 10 & $1.2 \mathrm{E}-01$ & 8.90 \\
\hline
\end{tabular}

Table 4: Test results of TSSA laminated connections - standard deviation and coefficient of variation

\begin{tabular}{ccccc}
\hline $\mathrm{T}$ & $\dot{d}$ & $\dot{\gamma}$ & $F_{V}$ & $F_{V}$ \\
{$\left[{ }^{\circ} \mathrm{C}\right]$} & {$[\mathrm{mm} / \mathrm{min}]$} & {$[-\mathrm{sec}]$} & St.dev $[\mathrm{kN}]$ & $\mathrm{COV}[-]$ \\
\hline-20 & 1 & $1.2 \mathrm{E}-02$ & 0.63 & 0.049 \\
23 & 1 & $1.2 \mathrm{E}-02$ & 0.61 & 0.059 \\
50 & 1 & $1.2 \mathrm{E}-02$ & 0.59 & 0.061 \\
80 & 1 & $1.2 \mathrm{E}-02$ & 0.52 & 0.064 \\
\hline
\end{tabular}

Figure 12 shows the shear resistance of SG connections plotted versus temperature and strain rate. The average values of resistance, displacement rate, strain rate and temperature are collected in Table 5. Table 6 provides then the standard deviation and the coefficient of variation for different temperatures. It is observed that the shear resistance of SG connections is much more sensitive to temperature variation and that it follows a 's-shape' behaviour. At the beginning, starting from $-20^{\circ} \mathrm{C}$ and increasing the temperature, the shear resistance exhibits a small negative gradient up to room temperature. Then, it shows an steep drop around $40^{\circ} \mathrm{C}$. Finally, it stabilizes again to small gradient at high temperature. The variation of strain rate has also effects and that the resistance seems to follow a logarithmic law. The temperature effects are much more pronounced than the strain rate effects. The SG standard deviation is variable with the temperature. The scatter of the response appears to be moderate and, compared to the $23^{\circ} \mathrm{C}$ one, slightly decreases at high and low temperatures (see Table 6). The coefficient of variation goes from $2 \%$ to $5 \%$.

(a)

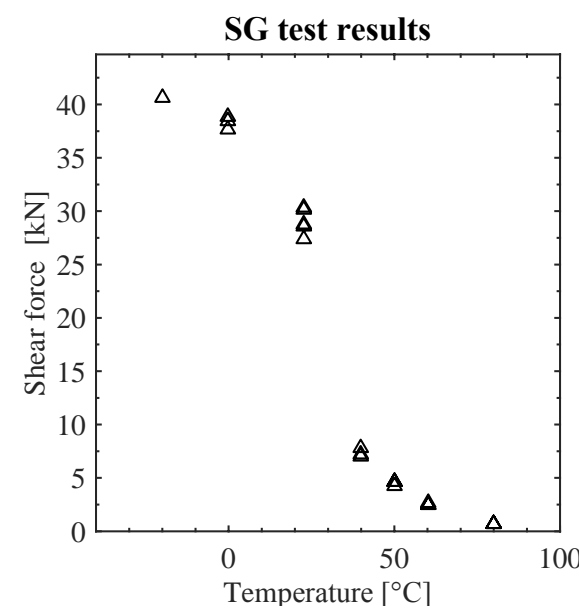

(b)

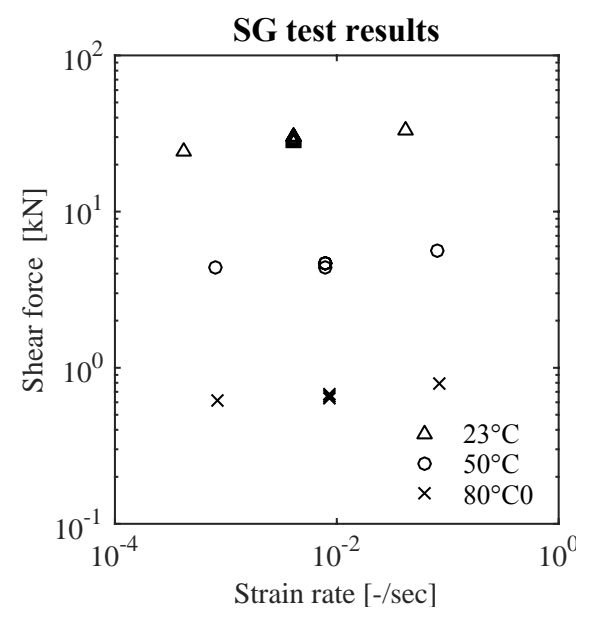

Figure 12: TSSA test results plotted versus (a) temperature (b) strain rate 
When tested at room and low temperature, the SG laminated connections always exhibit adherend failure, namely glass failure. In particular crack through thickness is occurring. It should be noticed that $19 \mathrm{~mm}$ fully tempered glass is used in this case. Different considerations can be made on this observation.

The first consideration is that the glass resistance of tempered glass might be not isotropic, i.e. not the same in all directions. Consequently, the value of tempered glass resistance provided by standards should be used with caution when the principal tensile stress is not laying along the in-plane direction (here indicated as $\mathrm{y}$-direction and $\mathrm{x}$-direction). This effect could be explained as follows. The tempering residual stresses, which are the cause of higher resistance of tempered glass, are the results of differential cooling times between surface and inner material. During the cooling process, the solidified material at the surface tends to constrain the in-plane contraction of the inner material (along $\mathrm{x}$ and $\mathrm{y}$ directions) causing the typical parabolic profile of compression-tensile-compression residual stresses. However, given the geometry of the problem, the material is free to contract out-of-plane (along z-direction). Therefore, limited residual stresses are built up along z-direction causing the nonisotropy of the residual stresses. The benefit of residual stress might be therefore very limited along zdirection. This consideration is of crucial importance with adhesive joints because the vectors of principal tensile stresses are often not oriented along $\mathrm{x}$-axis and $\mathrm{y}$-axis.

The second consideration regards the stable crack propagation. It is observed that, conversely to the expectation, it is possible to induce large stable cracks in tempered glass, without explosive glass failure. A possible interpretation of this behaviour is the following. Once the crack is initiated at the glass surface it starts propagating through the thickness of the glass panel. The crack initiates with an angle not perpendicular to the glass surface due to shear and residual stress. Then, within the material, the component of residual stresses orthogonal to the crack plane tends to close the crack, thus to slow it down and deviate its propagation direction towards the compressive principal direction. Subsequently, during this propagation, if the crack reaches the inner tensile zone of tempering profile, the crack propagates in a typical explosive manner. Otherwise, the crack continue to propagate downwards in a stable manner and goes back again to the surface. An interesting observation is that during this phenomenon, neither loss of rigidity nor loss of force is measured during the test. This might be related favourable contribution of the adhesive that, at room and low temperature, is rigid enough and resistant to act as a bridge between the crack and uncracked material.

Further considerations on the stress state in the laminated connections and on the shape of the crack will be provided in the following section.

The critical depth of stable cracking is observed to be around $20 \%$ of the thickness. This qualitative observation is confirmed by the measures of the residual stresses performed by means of a scattered light polariscope (SCALP-05). The stress profile is given in Appendix C. 
Table 5: Test results of SG laminated connections - mean values

\begin{tabular}{cccc}
\hline $\mathrm{T}$ & $\dot{d}$ & $\dot{\gamma}$ & $F_{V}$ \\
{$\left[{ }^{\circ} \mathrm{C}\right]$} & {$[\mathrm{mm} / \mathrm{min}]$} & {$[-/ \mathrm{sec}]$} & {$[\mathrm{kN}]$} \\
\hline$-20^{*}$ & 1 & $3.9 \mathrm{E}-03$ & 41.20 \\
0 & 1 & $4.0 \mathrm{E}-03$ & 38.29 \\
23 & 0.1 & $4.1 \mathrm{E}-04$ & 23.92 \\
23 & 1 & $4.1 \mathrm{E}-03$ & 29.00 \\
23 & 10 & $4.1 \mathrm{E}-02$ & 32.69 \\
40 & 1 & $7.0 \mathrm{E}-03$ & 7.37 \\
50 & 0.1 & $7.9 \mathrm{E}-04$ & 4.41 \\
50 & 1 & $7.9 \mathrm{E}-03$ & 4.52 \\
50 & 10 & $7.9 \mathrm{E}-02$ & 5.64 \\
60 & 1 & $8.2 \mathrm{E}-03$ & 2.56 \\
80 & 0.1 & $8.5 \mathrm{E}-04$ & 0.61 \\
80 & 1 & $8.5 \mathrm{E}-03$ & 0.65 \\
80 & 10 & $8.5 \mathrm{E}-02$ & 0.80 \\
\hline
\end{tabular}

* Machine limit is reached before failure for 2 specimens

Table 6: Test results of SG laminated connections - standard deviation and coefficient of variation

\begin{tabular}{ccccc}
\hline $\begin{array}{c}\mathrm{T} \\
{\left[{ }^{\circ} \mathrm{C}\right]}\end{array}$ & $\begin{array}{c}\dot{d} \\
{[\mathrm{~mm} / \mathrm{min}]}\end{array}$ & $\begin{array}{c}\dot{\gamma} \\
{[-\mathrm{sec}]}\end{array}$ & $\begin{array}{c}F_{V} \\
\text { St.dev }[\mathrm{kN}]\end{array}$ & $\begin{array}{c}F_{V} \\
\text { COV [-] }\end{array}$ \\
\hline 0 & 1 & $4.0 \mathrm{E}-03$ & 0.54 & 0.014 \\
23 & 1 & $4.1 \mathrm{E}-03$ & 1.20 & 0.041 \\
40 & 1 & $7.0 \mathrm{E}-03$ & 0.35 & 0.048 \\
50 & 1 & $7.9 \mathrm{E}-03$ & 0.15 & 0.034 \\
60 & 1 & $8.2 \mathrm{E}-03$ & 0.10 & 0.039 \\
80 & 1 & $8.5 \mathrm{E}-03$ & 0.02 & 0.028 \\
\hline
\end{tabular}




\section{Numerical analysis}

In the following sections the shear test of laminated connections is analysed by means of finite element model. The goal of this FEM analyses is to study the mechanical behaviour of the laminated connections by a numerical model able to accurately compute the stress distribution accounting for the three dimensional effects of the problem and real boundary conditions. More specifically, the aims are to (i) study the non-linear stress distribution in the adhesive (ii) evaluate the effects of adhesive stiffness on the stress distribution by parametrical analysis (iii) quantify the stress peak occurring in the adhesive and (iv) validate the three-dimensional stress distribution with an experimental observation of glass fracture during the test.

\subsection{Model geometry, boundary conditions and meshing}

A three-dimensional finite element model of the laminated connection is realized with software ABAQUS. Figure 13 shows the model geometry, dimensions and boundary conditions. The thickness of the adhesive in the model is $1.5 \mathrm{~mm}$ for SG and $1 \mathrm{~mm}$ for TSSA. The glass thickness is $19 \mathrm{~mm}$ for SG and $15 \mathrm{~mm}$ for TSSA. Only half of the connection is implemented making use of the symmetry along y-axis. The SG adhesive is modelled with elasto-plastic material and the TSSA adhesive is modelled with hyper-elastic material. Material inputs are taken from [12] at similar strain rate as occuring in this work. SG plastic strain curve are taken from [12] and the Mooney-Rivlin model is used for TSSA. Quasi-static step-by-step analyses are performed using iterative implicit solver algorithm. Finite deformation theory is used to account for geometrical non-linearity and large deformations.

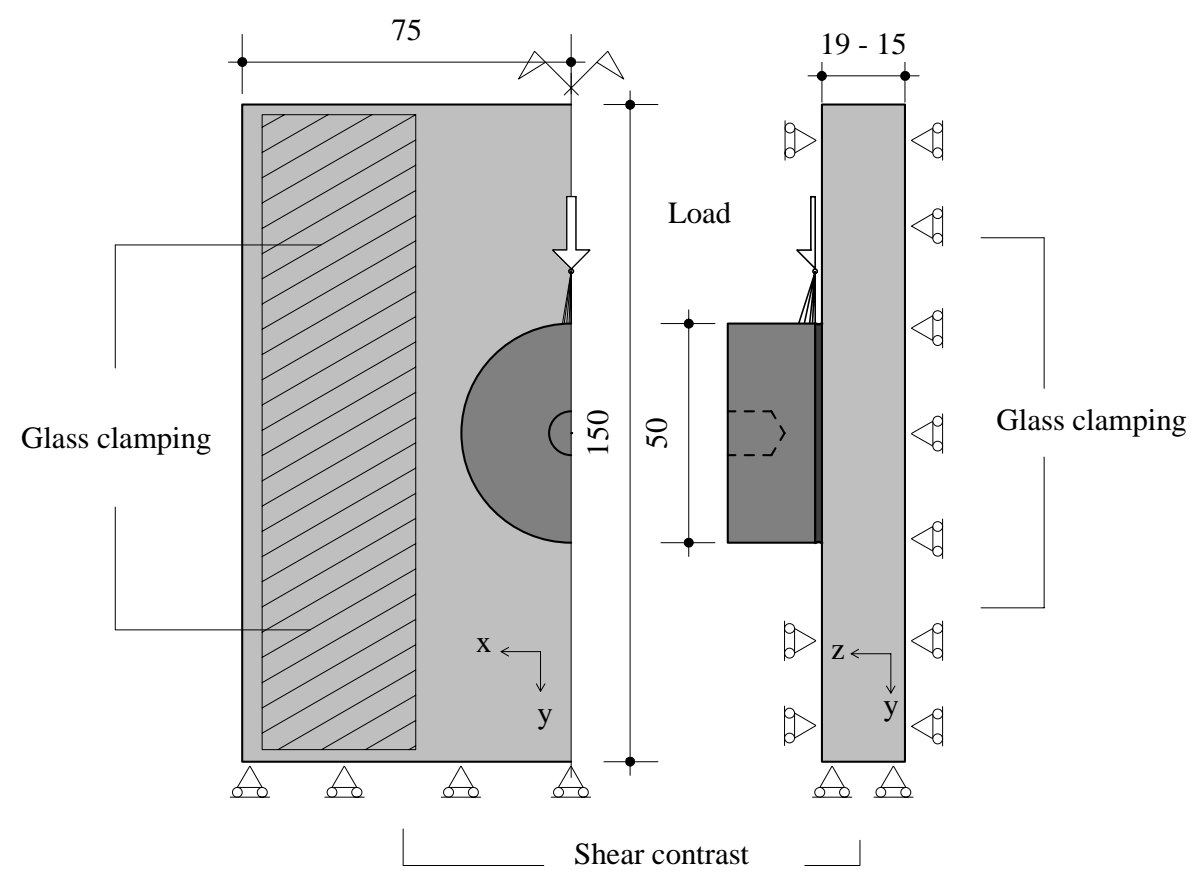

Figure 13: Geometry, dimensions and boundary conditions of the numerical model

The boundary conditions and load application are implemented as close as possible to the testing setup. The horizontal displacements of the glass panel are constrained at the glass surface, as indicated in Figure 13 and described in the experimental part. The vertical displacements of the glass panel are constrained at the lower edge. The shear load is applied to the metal connector as close as possible to the adhesive, as it is done in the tests. To do so, a multiple-points constrain interaction is built between 
a reference point and the metal connector at the load introduction region. The shear load is then applied to the reference point that redistributes the force to the metal connector.

The results of mesh and elements study indicate tetrahedral elements as an optimal choice for this problem in term of result accuracy and computational cost. The details of solver, element and mesh study are given in Appendix B. Figure 14 and Figure 15 show the mesh of the model. The mesh is strongly refined at the level of the adhesive (see Figure 15 (c)). Large stress gradients are indeed expected to occur in that region. For this reason, the mesh is also refined at the free edge of the adhesive itself (see Figure 15 (d) and (b)). The mesh size at the adhesive is no larger then $0.2 \mathrm{~mm}$. Second order elements are usually used. When convergency cannot be achieved, usually in the case of large deformations, the mesh size is further reduced and first order elements are used to achieve convergency. Control of the pressure profiles is performed to ensure that volumetric locking is not occurring. All simulations show continuous pressure profiles. The use of elements with modified or hybrid formulation was therefore not required. Full precision is used because a large number of iterations is required to achieve the solution of the problem.

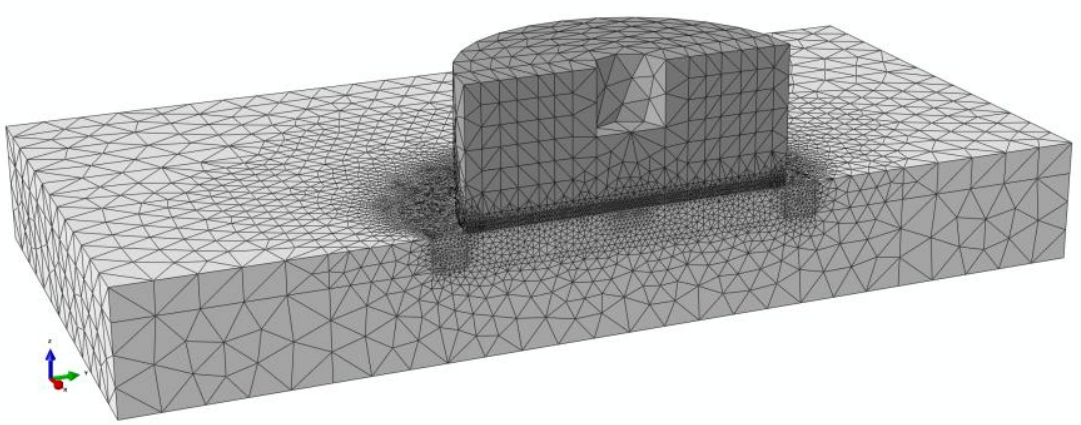

Figure 14: Mesh of the three-dimensional model for shear test - 3D view. In light grey is the glass, middle grey is the steel and dark grey is the adhesive

(a)

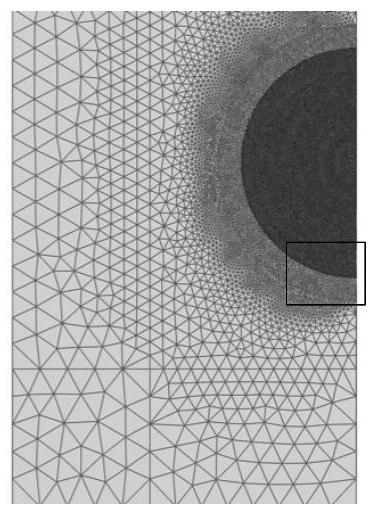

(b)

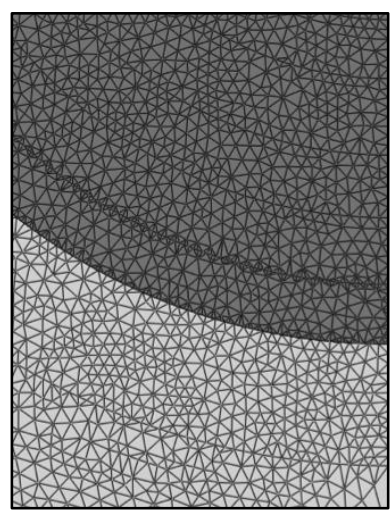

(c)

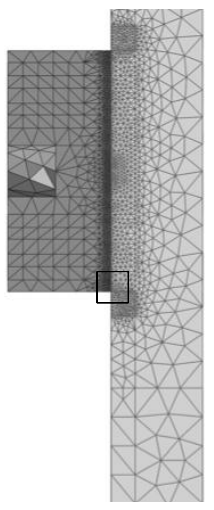

(d)

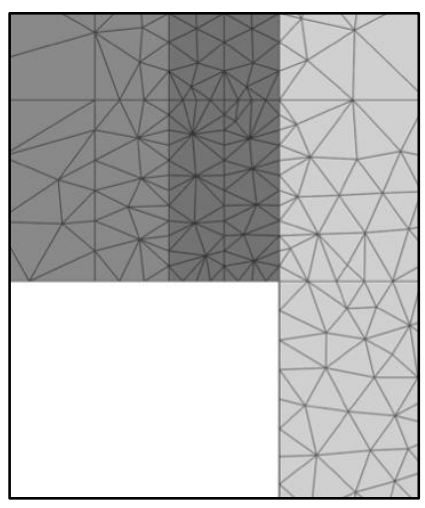

Figure 15: Mesh of the three-dimensional numerical model: (a-b) plan views (c-d) lateral views. Light grey is glass, middle grey is steel and dark grey is adhesive

\subsection{Discussion of numerical results}

Figure 16 shows the field distribution of shear stresses, $\tau_{y z}$, in laminated connections under shear load. The stress distribution in the connection is non-uniform along $\mathrm{x}$-axis, $\mathrm{y}$-axis and z-axis, see Figure 16 (a) and Figure 16 (b). The non-uniformity of the stress distribution along $x$-axis and $y$-axis is because 
the load is gradually transferred from the circular metal connector into the glass panel, and then transferred to the bottom glass edge, which is linearly constrained against vertical displacement. The non-uniform stress distribution along z-axis, i.e. through thickness, is because the thicknesses of the adherends are much larger than the adhesive one. Because of this difference, the adhesive is not able, stress-wise, to fully involve the adherends through thickness. The stresses in the adherends are thus mainly localized close to the adhesive. Because of these reasons, the stress distribution is dependent on the three-dimensional connection geometry and on the boundary condition of the experimental testing.

(a)

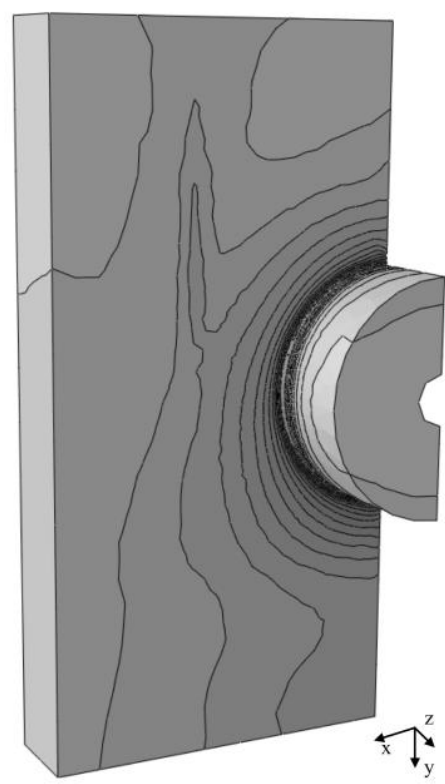

(b)

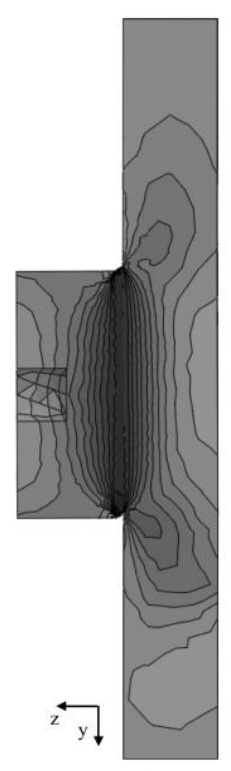

(c)

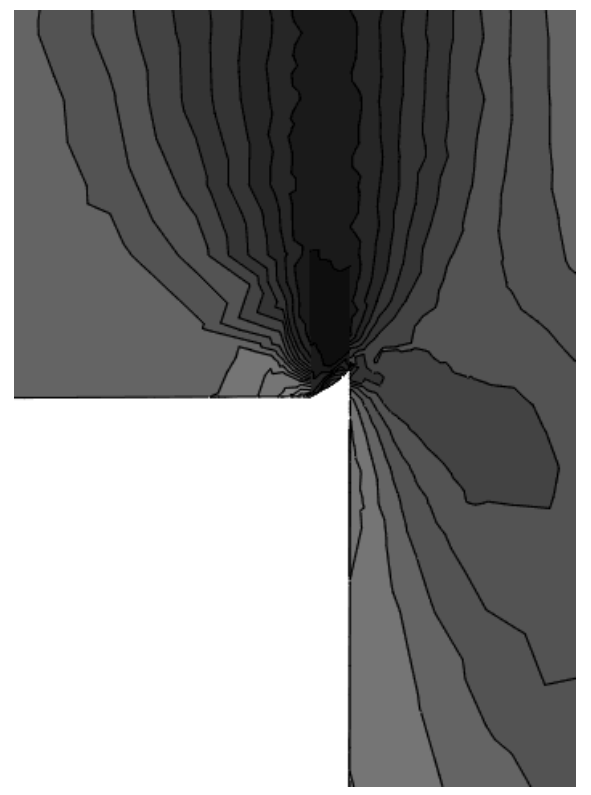

Figure 16: Shear stresses $\tau_{y z}$ distribution in laminated connections under shear force (a) $3 \mathrm{~d}$ view (b) lateral view and (c) zoom of the lateral view at the adhesive

The field distribution of the shear stresses in the connection shows intensification at the level of the adhesive (see Figure 16 (b)). Within the adhesive, maximum shear stresses are occurring at the adhesive adherend interfaces. In particular, the maximum stress peaks occur close to the free edge of the adhesive (see Figure 16 (c)). Far from the edges, the shear stress distribution in the adhesive is rather uniform. A parametrical numerical study is performed in the following section to quantify the value of the stress peaks occurring in the adhesive. A simplified analytical variational approach is then used in section 4 to understand the physics of this peaks and to derive analytically how different parameters influence the stress distribution.

In brittle materials like glass, the initiation of crack is usually dominated by tensile stresses and the crack plane tends to be located orthogonally to the principle (tensile) stresses. The crack pattern is therefore expected to follow the direction of minimal principal stresses that are, by definition, orthogonal to the maximum principal stress. The stress distribution of the minimal principal stress obtained from the numerical model is shown by Figure 17 (a) and by Figure 17 (b). Figure 17 (c) and Figure 17 (d) show instead the experimental observation of the stable glass cracking through thickness. It can be observed that the numerical model shows good correspondence with the experimental observation. The stress field computed with the numerical model through thickness shows a 'drop-like' shape. The latter is also observed in the experimental observations. Furthermore, the predicted location of this crack is also confirmed by the test. Indeed, the crack initiates at approximately half of the diameter (middle of the connection along y-axis) as indicated by the numerical modelling (see Figure 17 (b)). 
(a)

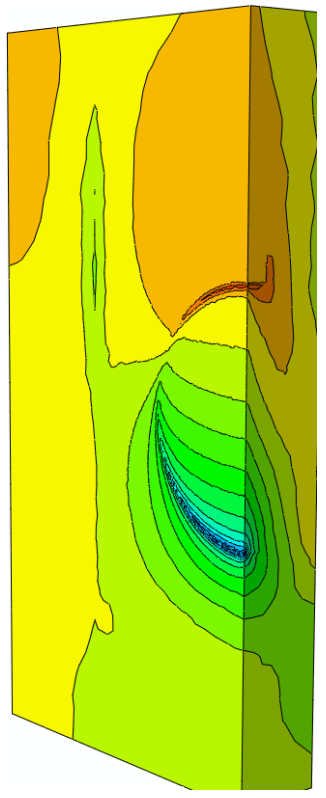

(b)

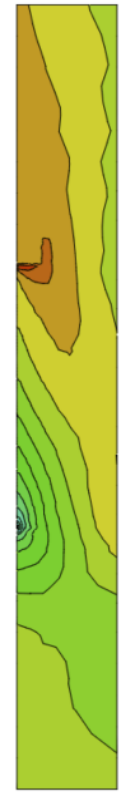

(c)

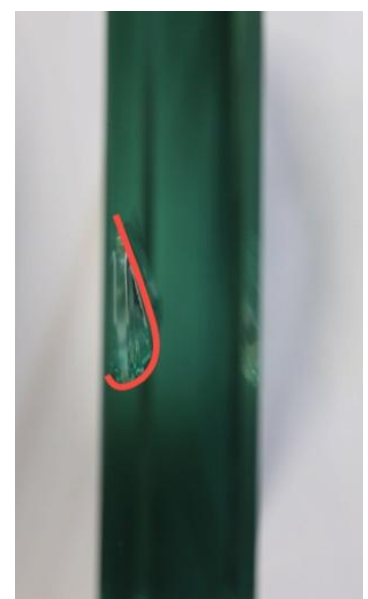

(d)

Figure 17: (a) 3D view and (b) through thickness view of the stress field distribution of minimal principal stress in the glass (the lines represent the section of iso-stress surfaces of the minimal principal component of the stress tensor) (c) experimental observation of drop-like stable crack in the glass panel (lateral view), crack profile indicated by red line (d) zoomed photo

Similar considerations can be made observing the frontal view of the glass panel. Figure 18 (a) shows the pattern of glass breakage after testing in case of not explosive glass failure. This is compared with the stress field distribution given by the numerical model (Figure 18 (b)). The numerical model gives stress field distribution that starts from the middle of the connection and propagates over the glass panel downward, similarly to the experimental observations.

(a)

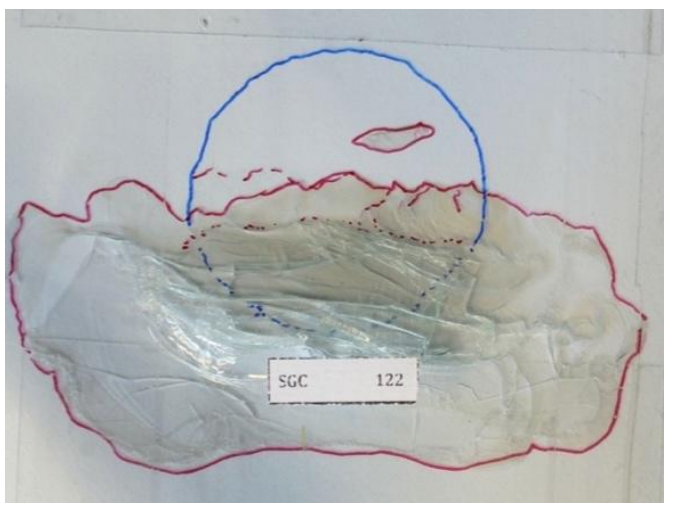

(b)

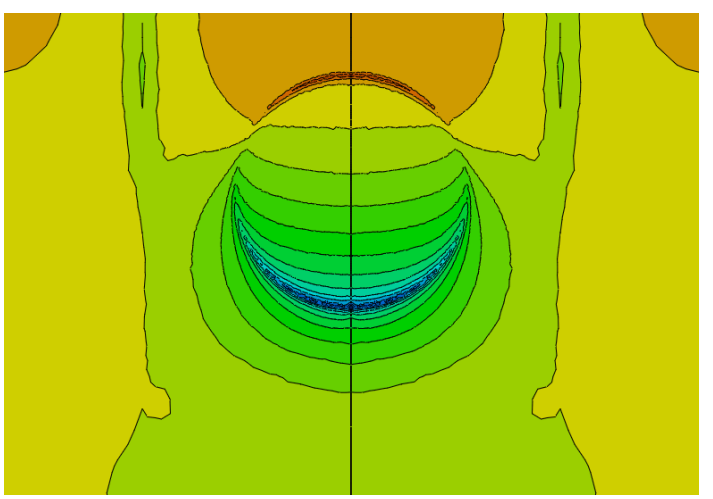

Figure 18: Glass breakage in laminated connections under shear load: (a) experimental results (b) stress field distribution of the minimal principal stresses computed with the numerical model

A further analysis of the crack pattern in the glass plate is now performed using a strut-and-tie approach. Figure 19 shows a simple two-dimensional model of the glass plate. The boundary conditions of the glass plate are assumed as in the numerical analysis. The shear force is applied to the metal connector, which is then transferred to the glass by the shear stresses of the adhesive layer (see Figure 19 (a)). The stress state in the glass is characterized by shear stress and compression vertical stress. This stress state corresponds to principal stresses inclined with respect to the vertical direction, 
as indicated by Figure 19 (c) $)^{11}$. Within the glass, compressed elements transfer then the load from the adhesive region to the glass edge at the bottom. A possible distribution of equilibrate compressed elements is given by Figure 19 (b). As mentioned before, the glass cracks are expected to occur orthogonal to the maximum (tensile) principal stresses. The crack is therefore expected to follow the distribution of the compressed elements of Figure 19 (b). The experimental observations appear to confirm the results of this analysis (see Figure 17 (c) and Figure 17 (d)).

(a)

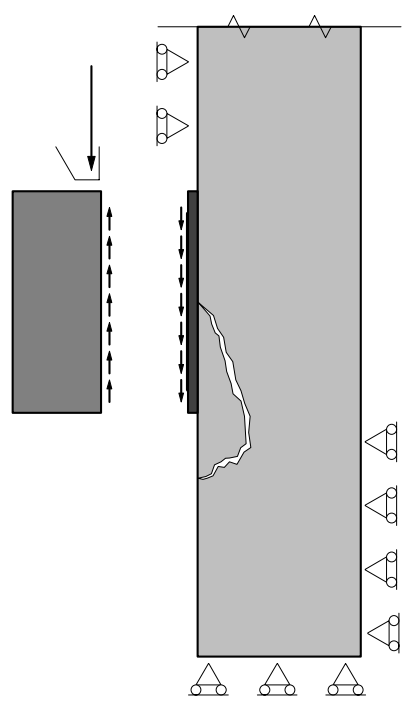

(b)

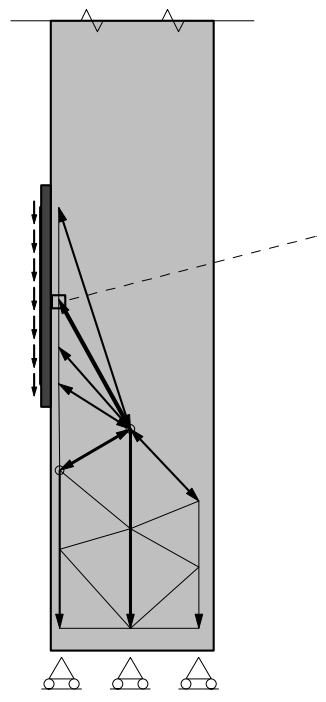

(c)

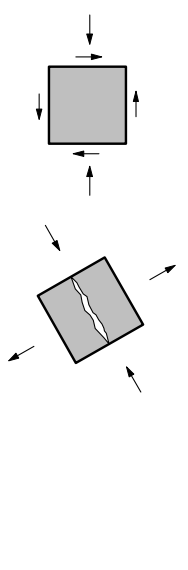

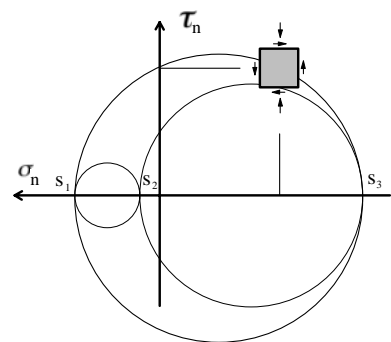

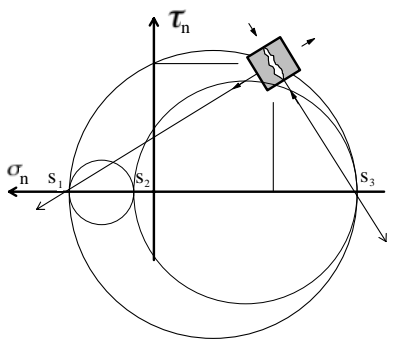

Figure 19: (a-b) Simplified forces distribution through thickness of the glass panel with compressed elements (c) Scheme of the stresses state in the glass panel at the adhesive: shear and normal stresses for different plan angle

\subsection{Parametrical study and stress factor definition}

In the previous section the stress distribution in the glass panel is analysed and compared with the experimental observations. In this section a parametrical study is performed varying the mechanical properties of the adhesive. The aims of this study are (i) to evaluate the behaviour of the stress distribution in the adhesive at different temperatures and (ii) to quantify the stress peaks as a function of the temperature. This is done by means of a factor, here called stress factor. The latter will be used in the final section for the derivation of a prediction model.

The parametrical study is performed by means of several numerical analyses carried out varying the stiffness of the adhesive. This is done to cover the full range of investigated temperature in the experimental campaign. The results of the parametrical study are plotted in Figure 20. The results are presented in terms of normalized stress versus normalized distance.

The normalized stress describes the non-linear stress distribution in the adhesive by the deviation of the actual stresses in the adhesive from the nominal one. Normalized stress is computed as the ratio between the actual stress in the adhesive, $\tau_{z y}(y)$, and the nominal stress, $\tau_{\text {nom }}$.

The actual stress is the one obtained from the non-linear three-dimensional FEM analysis ${ }^{12}$. The nominal stress is calculated as the applied shear force, $F_{V}$, divided by adhesive area, $A$. In Figure 20 (a), the distribution of the normalized stress is plotted against normalized distance. Normalized

\footnotetext{
${ }^{11}$ This simplified scheme represents, as a function of the angle, the stress state, i.e. the normal and total shear stresses acting over plane that contains the middle principal direction, the latter assumed to be constant and with direction along $\mathrm{x}$-axis

${ }^{12}$ The stresses are computed with applied load close to the failure load because the stress distribution depends also on the load level.
} 
distance is calculated as $y$-coordinate divided by the diameter of the metal connector, with zero at the center and one at the perimeter of the metal connector

$$
\alpha_{V}=\frac{\tau_{z y, \max }}{F_{V} / A}=F\left(E_{i}, t_{i}, B C s .\right)
$$

It is observed that the stress distribution is in general non-linear and shows peaks close to the free edges of the adhesive. The stress peaks vary with the stiffness of the adhesive, therefore with the temperature. The stress peaks are larger with stiff ${ }^{13}$ adhesives, i.e. SG at low temperatures, and lower with flexible adhesive, i.e. SG at high temperatures or TSSA. The results are then summarized by the definition of an adimensional stress factor, $\alpha_{V}$, which is calculated as the stress peak of the stress field distribution divided by the nominal stress (equation (1) ${ }^{14}$. Given the applied nominal stress, the stress factor allows to compute the stress peak occurring in the adhesive. The stress factors for SG and TSSA at different temperature are collected by Table 7 and plotted by Figure 20 (b). The SG stress factor shows inverse s-shape behaviour while the TSSA stress factor show negligible variations with the temperature.

Table 7 Shear stress factor for laminated connections under shear load at different temperatures

\begin{tabular}{ccccccccc}
\hline $\mathrm{T}\left[{ }^{\circ} \mathrm{C}\right]$ & 80 & 60 & 50 & 40 & 30 & 23 & 0 & -20 \\
\hline$\alpha_{V}$ for SG & 1.036 & 1.046 & 1.056 & 1.066 & 1.093 & 1.100 & 1.107 & 1.116 \\
$\alpha_{V}$ for TSSA & 1.041 & & 1.041 & & & 1.041 & & 1.041 \\
\hline
\end{tabular}

It should be noticed that the values of stress factor collected in Table 7 refer only to the geometry and boundary conditions investigated in this work. They should be used with caution, and in general not extended to different testing condition without further detailed studies.

(a)

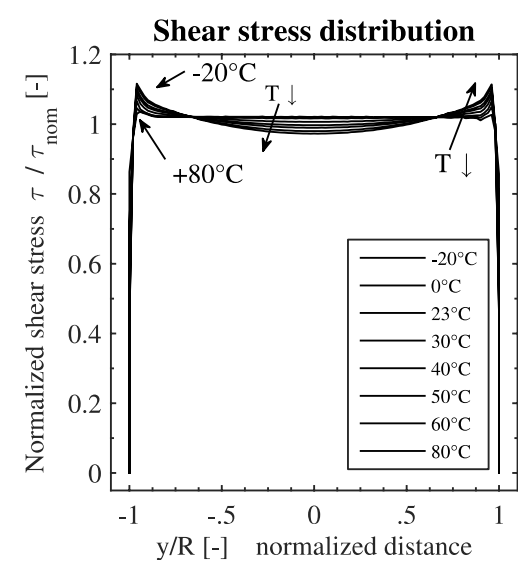

(b)

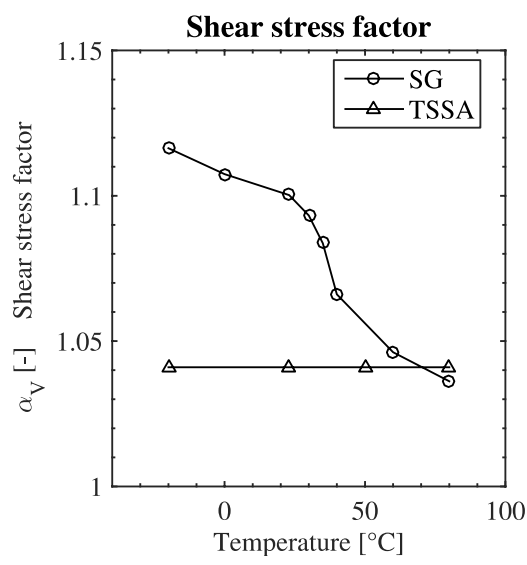

Figure 20: (a) Parametrical study of the shear stress distribution in the SG adhesive at varying temperature (b) Shear stress factor at different temperatures for laminated connections.

An additional consideration should be made on the stress state of the adhesive. Even when a pure shear load is applied to the connections, normal tensile and compressive stresses are occurring in addition to the shear. In the case of pure shear loading indeed, the eccentricity is reduced to the minimum but yet not zero, i.e. equal to the adhesive stiffness. These normal stresses can be high in the case of flexible adherends. Conversely, in cases very rigid adherends, like in the present work, these normal stresses are limited. However, they should be taken in consideration. More specifically, the three-dimensional stress state configuration of the stress tensor should be accounted. This is usually

\footnotetext{
${ }^{13}$ More specifically, the stress peaks are larger when the global adhesive stiffness, i.e. shear modulus divided by the thickness, is larger.

${ }^{14}$ The use of the stress factor together with the results of the experimental investigations allows the derivation of failure prediction models, which are described more in detail in the following section 5.
} 
done with a solid mechanics parameter known as triaxiality. This coefficient gives information on the ratio between hydrostatic and deviatoric components of the three-dimensional stress tensor. In pure shear loading, with rigid and constrained adherend, the triaxiality is limited yet not zero. The values of triaxiality for the shear tests presented in this work are given in Table 8. More information and analytical studies on this subject can be found in [71].

Table 8: Triaxiality in laminated connections under shear load at different temperatures

\begin{tabular}{ccccccccccc}
\hline Material & \multicolumn{1}{c}{ SG } & \multicolumn{1}{c}{ TSSA } \\
\hline $\mathrm{T}\left[{ }^{\circ} \mathrm{C}\right]$ & -20 & 0 & 23 & 30 & 40 & 50 & 60 & 80 & any \\
Triaxiality $\eta[-]$ & 0.08 & 0.08 & 0.08 & 0.09 & 0.10 & 0.14 & 0.18 & 0.34 & 0.23 \\
\hline
\end{tabular}




\section{Analytical study and simplified variational approach}

In the previous section it is observed that the stress distribution in laminated connections is in general non-uniform and varies over $\mathrm{x}-, \mathrm{y}$ - and $\mathrm{z}$-axes. It is shown that, to accurately compute the stress distribution and the stress peaks, accurate numerical models, that permit to properly account for the three-dimensionality of geometry and boundary conditions, are required.

However, analytical approaches, even though simplified, are also of interest because they help in analysing the physical phenomenon behind the mechanical behaviour of structural component. Because of that, in this section, the problem is analysed by a simplified two-dimensional analytical variational approach. It should be noticed that the aim of this study is not to precisely compute the values of the stress peaks, because, as it is shown in the previous section, accurate three-dimensional numerical analyses are needed. The goals are instead to (i) understand the physic behind the nonlinearity of the stress distribution in the adhesive (ii) analytically derive the parameter on which the stress distribution depend on and (iii) evaluate the effects of the parameters variations by means of analytical parametric study.
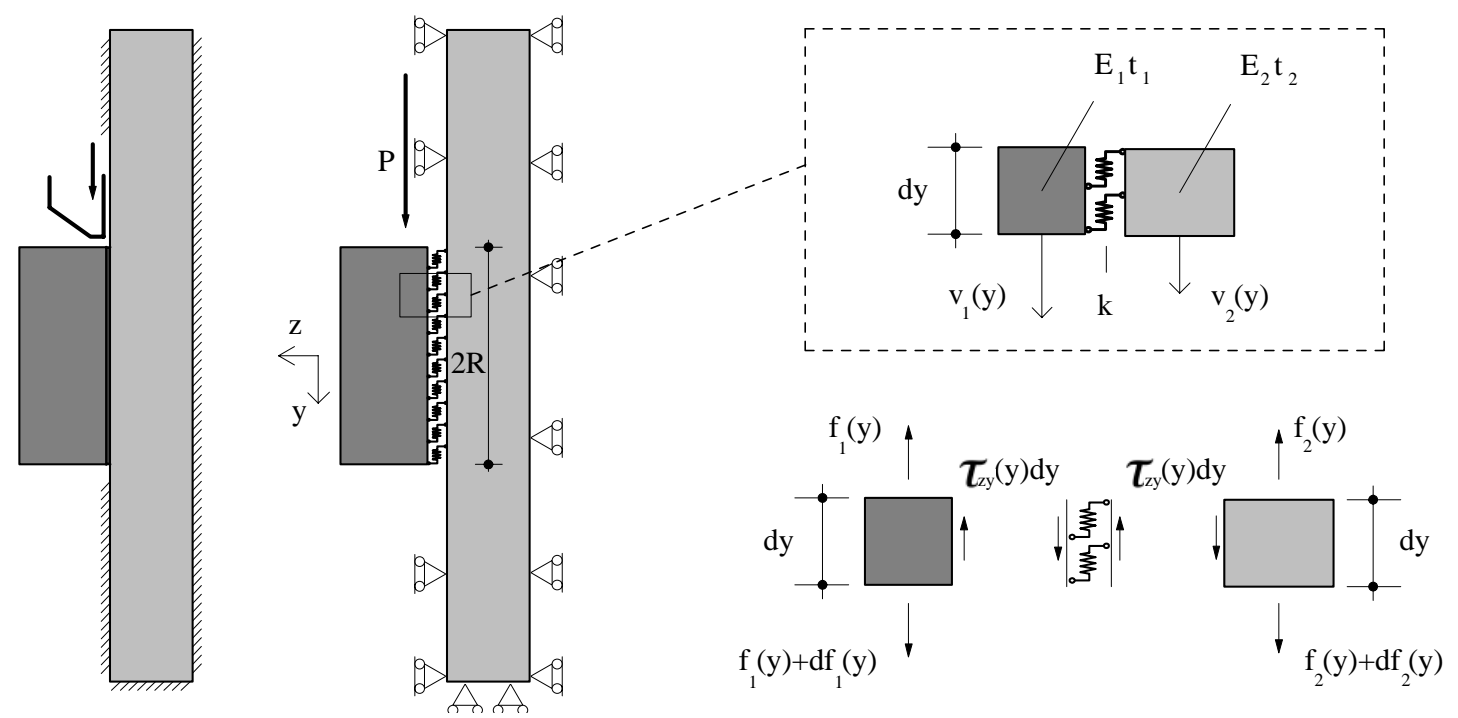

Figure 21: Scheme of the analytical model

Figure 21 shows the scheme of the simplified two-dimensional spring model used in this study. Boundary conditions are assumed to be the same of the numerical model. The $x-y-z$ reference system is defined with the $x-y$ plane at the adhesive layer, $z$-axis orthogonal to the glass plate and $y$-axis along the loading direction. Positive y coordinates are downwards with zero value in the middle of the connection. The adhesive layer between the two adherends, glass plate and steel connector, is modelled with a bed of infinitesimal mono-dimensional equivalent shear springs. The rigidity of these infinitesimal springs is here indicated with $k$ [FL^-3]. The parameter $k$ is assumed to be constant for each point of the adhesive over y axis. The two adherends are generally characterized by different Young's modulus and thickness, $E_{1}$ and $t_{1}$ for the metal and $E_{2}$ and $t_{2}$ for the glass plate.

The vertical displacement of the adherends is indicated with $v_{1}(y)$ for the metal connector and $v_{2}(y)$ for the glass plate. The constitutive law of the springs is given by (2), where $\tau_{z y}(y)$ [FL^-2] is the shear force in the adhesive, and $\delta(y)$ is the relative displacement between the adherends.

$$
\begin{gathered}
\tau_{z y}(y)=k \cdot\left[v_{1}(y)-v_{2}(y)\right]=k \cdot \delta(y) \\
\text { with } \quad k=\frac{E_{a d h}}{2(1+v) \cdot t_{a d h}}
\end{gathered}
$$


Let us now consider an infinitesimal portion of the connection of length $d y$. This creates two infinitesimal portion of adherends, see Figure 21-right. The dark grey element represents the metal and the light grey represents the glass. Because of the relative displacement between the two adherends, the spring applies two shear forces on the metal and the glass, with equal amplitude, $\tau_{z y}(y) d y$, and opposite direction. The normal forces acting in the two adherends are indicated with $f_{l}(y)$ for the metal and $f_{2}(y)$ and for the glass adherend. The forces in the adherend are in general variable over the y-axis and the variation over an infinitesimal length is indicated as $d f(y)$. The equilibrium of forces must be satisfied for both infinitesimal adherends. This leads to equation (3) and equation (4).

$$
\begin{aligned}
& d f_{1}(y)-\tau_{z y}(y) d y=0 ; \quad \frac{d f_{1}(y)}{d y}=\tau_{z y}(y) \\
& d f_{2}(y)+\tau_{z y}(y) d y=0 ; \quad \frac{d f_{2}(y)}{d y}=-\tau_{z y}(y)
\end{aligned}
$$

The constitutive laws of the adherends are given by equation (5), written in the variational form of the vertical displacements. The derivation of relative displacement with respect to $\mathrm{y}$, accounting for equation (5), leads to equation (6).

$$
\begin{gathered}
\epsilon_{1}(y)=\frac{d v_{1}(y)}{d y}=\frac{f_{1}(y)}{E_{1} t_{1}} ; \quad \epsilon_{2}(y)=\frac{d v_{2}(y)}{d y}=\frac{f_{2}(y)}{E_{2} t_{2}} \\
\frac{d \delta(y)}{d y}=\left(\frac{f_{1}(y)}{E_{1} t_{1}}-\frac{f_{2}(y)}{E_{2} t_{2}}\right) \\
\frac{d^{2} \delta(y)}{d y^{2}}=\left(\frac{\tau_{z y}(y)}{E_{1} t_{1}}+\frac{\tau_{z y}(y)}{E_{2} t_{2}}\right)=\delta(y) \cdot k \cdot\left(\frac{1}{E_{1} t_{1}}+\frac{1}{E_{2} t_{2}}\right)
\end{gathered}
$$

Equation (6) is then derived with respect to y accounting first for equations (3) and (4) and then equation (2). The final differential equation (8), that governs the problem, is therefore achieved. This governing expression is a second-order, homogeneous, differential equation, with constant parameters. The parameter $\phi$, here called as relative stiffness factor, is a function of the material properties, geometry and the spring stiffness.

$$
\begin{gathered}
\frac{d^{2} \delta(y)}{d y^{2}}-\phi^{2} \delta(y)=0 \\
\text { with } \phi=\sqrt{k \cdot\left(\frac{1}{E_{1} t_{1}}+\frac{1}{E_{2} t_{2}}\right)}
\end{gathered}
$$

The solution of the homogeneous differential equation (8) has the form of equation (9). The coefficients $A$ and $B$ can be derived by considering the boundary conditions of the problem. Firstly, with $R$ being the connector radius, the stress field is assumed to be symmetric with respect to the center (i.e. $f(R)=f(-R))^{15}$, and with equation (10) $A=B$ is achieved. Then, the global equilibrium with the external forces is imposed by equation (11) and the coefficients A and B are derived.

$$
\begin{gathered}
\delta(y)=A e^{-\phi y}+B e^{+\phi y} \\
A e^{-\phi R}+B e^{+\phi R}=A e^{+\phi R}+B e^{-\phi R} \rightarrow A=B \\
\int_{-R}^{R} f(y) d y=\int_{-R}^{R} k \delta(y) d y=A \frac{2 k}{\phi}\left(e^{+\phi R}-e^{-\phi R}\right)=P
\end{gathered}
$$

\footnotetext{
${ }^{15}$ This approximation is valid in case of the two adherend have similar stiffness
} 


$$
A=B=\frac{P \phi}{2 k}\left(e^{+\phi R}-e^{-\phi R}\right)^{-1}
$$

The combination of equation (11) with equations (9) and (2) gives equation (12). The latter is the mathematical expression that describes the stress distribution, $\tau_{z y}(y)$, in the adhesive as a function of the position $y$. This equation confirm the expressions derived by Volkersen [72].

$$
\tau_{z y}(y)=\frac{P \phi}{2} \frac{\left(e^{-\phi y}+e^{+\phi y}\right)}{\left(e^{+\phi R}-e^{-\phi R}\right)}
$$

From equation (12) it is obtained that the stress distribution is non linear and that the stress distribution depends on several parameters. More specifically, it is derived that the stress distribution and the stress peaks are function of the stiffness of the spring, the stiffness of the adherends, the thickness of the adherends and the overlapping length, which in the current problem is the metal connector radius $R$. The shear stresses are in general lower than the nominal one in the middle of the connection and larger towards the free edges of the adhesive.

A parametrical study is now performed to analyse the effects of these parameters on the stress distribution. Two groups of analyses are carried out. The first set of analyses is done to investigate the effects of the adhesive stiffness, adherend stiffness and thicknesses. The second set of analyses is to investigate the effects of radius. The results are presented as stress factor versus normalized distance. Normalized distance is calculated as the distance from the middle of the connection divided by the radius, being therefore zero at the center and one at the free edge. The stress factor is the same defined in the previous section, i.e. it describes the deviation of the actual stress from the nominal one.

Figure 22 shows the results of the first set of parametric analyses, i.e. the stress distribution in the adhesive with different values of the relative stiffness factor and connector radius of $25 \mathrm{~mm}$. Equation (12) and Figure 22 show that the shear stress distribution exhibits peaks close to the edges (normalized distance equal to one) and that these peaks increase exponentially when the adhesive stiffness increases. Conversely, when the adhesive stiffness is much smaller than the adherend one, the stress distribution tends to be uniform. These observations are in line with the numerical results presented in the previous section.

Figure 23 shows the results of the second set of parametric analyses, i.e. the stress distribution in the adhesive for different radius. The same group of analyses is repeated for three values of relative stiffness factor. Among others, the main finding here is that the relative efficiency of the joint decreases with increasing radius (see Figure 23 (a)). This is because the shear force is mainly transferred with shear stresses concentrated in a region close to the end of the joint. Far from the edges, i.e. normalized distance going toward zero, the shear stresses decreases rapidly. In case of large radius, the portion of joint far from the edge is not contributing significantly to the shear load transfer. This means that the increase of radius, after a certain value, is not an efficient action to increase the global resistance of the joint. This effect is more pronounced with stiff adhesive and tends to zero with very soft adhesive, where the stress distribution tends to be uniform (see Figure 23 (b) and (c)). 
(a)

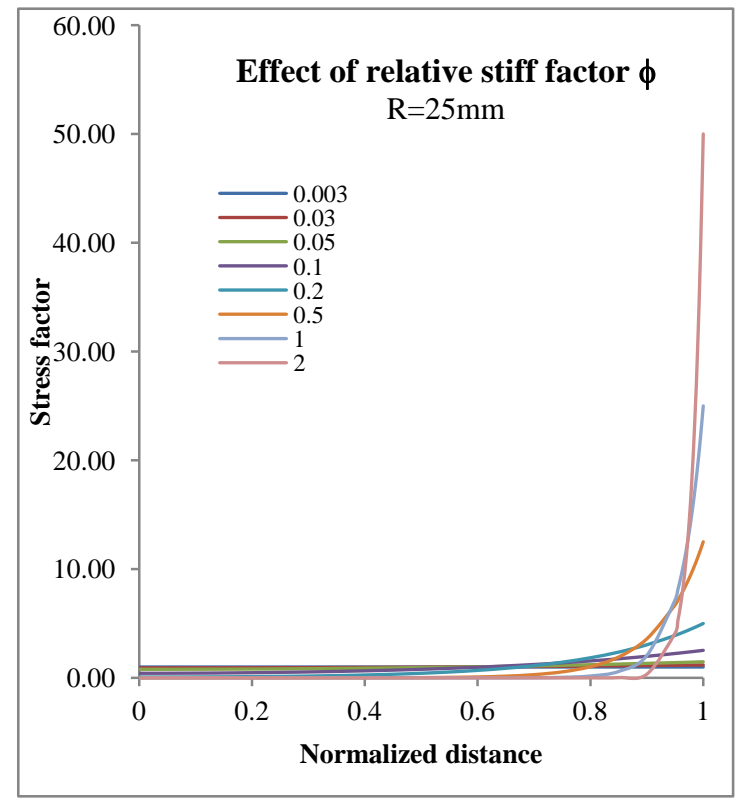

(b)

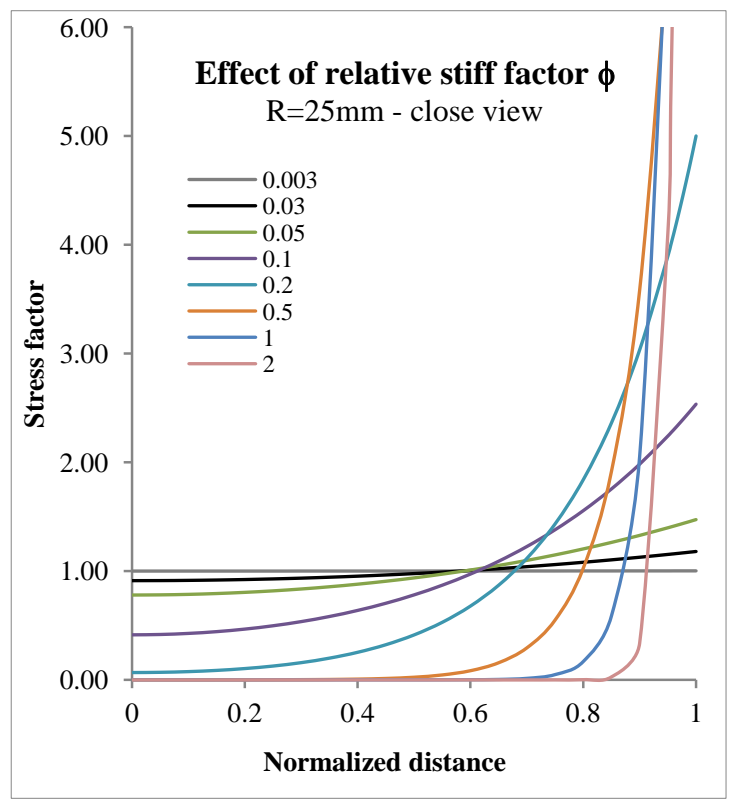

Figure 22. Analytic parametric study: effects of the relative stiffness factor on the stress distribution and stress peaks (a) zoomed view on a reduced stress factor scale

(a)

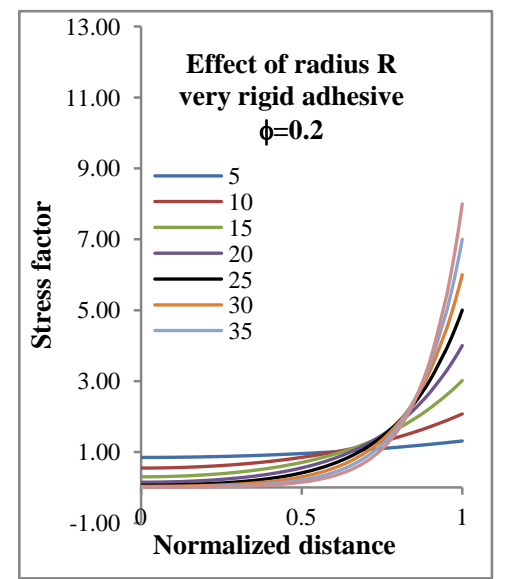

(b)

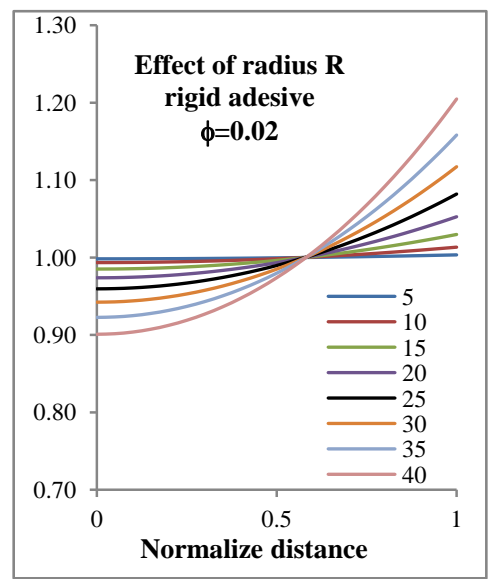

(c)

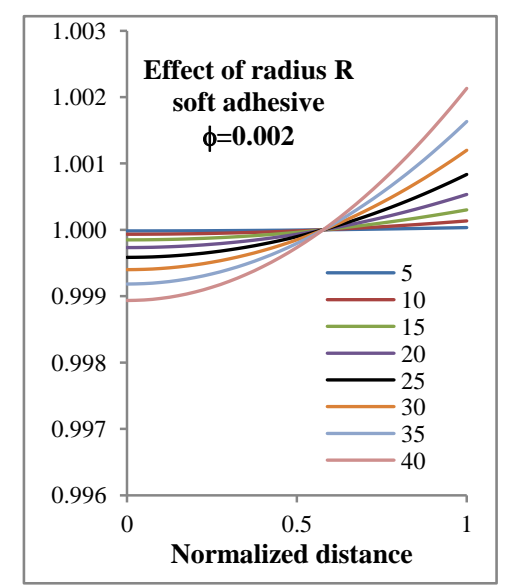

Figure 23. Analytic parametric study: effects of the radius on the stress distribution and stress peak for different value of the relative stiffness factor: (a) very rigid (b) rigid and (c) soft adhesive

It should be noticed that this simplified approach does not consider the three-dimensionality of the current problem, which requires the use of numerical analysis for the accurate computation of the stress peaks. In that regards different considerations should be made.

A first consideration is that the analytical expressions derived in this approach consider constant stress state along $\mathrm{x}$-axis and $\mathrm{z}$-axis. However, the numerical results and experimental observations discussed in the previous sections show that the stress distribution is in general non-uniform. Indeed, it is firstly observed that the stresses in the glass vary along $\mathrm{x}$-axis. This is because the stresses gradually diffuse from the connector to the bottom edge of the glass. Secondly, it is also observed that the stresses are also not uniform through thickness $\left(\mathrm{z}\right.$-axis ${ }^{16}$ ). This is because, with the geometry of the current problem, the adhesive is not able to involve the full thickness of the adherends.

\footnotetext{
${ }^{16}$ The hypothesis of constant adherend stress through thickness also creates non-zero stress at the edge of the adhesive. This is in contrast with the condition of rotational equilibrium of tangential stresses at the free edge of the adhesive.
} 
A second consideration is on the geometry of the connection. More specifically, it concerns the hypothesis of constant stiffness over the y-coordinates. The circular geometry of the metal connection is indeed not accounted by this approach. The circular geometry causes a not constant width ( $\mathrm{x}$ direction) of the metal connector and of the adhesive along y-coordinate. It follows that the relative stiffness factor, $\phi(y)$, is not constant along y-axis. This could be accounted for by deriving the expression of the width as a function of $y$, calculating the stiffness as a function of $y$ and accounting for this variability ${ }^{17}$ in the derivation of the final differential equation. This would lead to a second order, homogeneous, differential equation with non-constant coefficients, which integration is more analytical complex than the equation (8). Because of these reasons, for the current problem, finite element analyses are therefore preferred for the accurate computation of the value of the stress peaks.

For different type of problems, more complex variational models can be used, which are able to capture additional phenomena. For instance, in the case of single-lap joint with very flexible adherends, cylindrical bending of the adherends is occurring if the out of plane displacements are not constrained. This deformability induces non-negligible bending moment in the adherends. In this case, the variational approaches should be extended to account also for the bending deformation of very flexible adherends. This leads to differential equations with higher order than the one here derived in equation (8). For example, in the approach proposed by [73], the governing equation is a seven-order differential equation. In such cases, the differential equation often requires to be numerically solved with computer, loosing the advantages of simple analytical explicit solutions. These methods were largely used when nonlinear finite elements were not advanced and efficient yet. However, given the capability and computational efficiency of their algorithms, current numerical finite element methods appear to gain large competitiveness against analytical one. This method allows to capture complex three-dimensional behaviours with reduced computational cost than analytical one. Furthermore, they can be used for any generic geometry with less difficulty than the analytical approach. For these reasons, in the case of geometrically complex problem such as the one of the present work, non-linear finite element analysis appears to be a preferred and most efficient approach for the computation of stress field distribution.

\footnotetext{
${ }^{17}$ For example the spring stiffness would assume the form of $\left.k(y)=\left(R^{2}-y^{2}\right)^{0.5} G / t / R\right)$, and the metal connector stiffness would be calculated by $\left.\left(\left(R^{2}-y^{2}\right)^{0.5} E t\right) / R\right)$
} 


\section{Proposed failure prediction model}

A general failure model that accounts for non-linear stress distribution, boundary conditions, geometry, temperature dependent behaviour and strain rate dependent behaviour is here proposed by equation (13). In equation (13), $F_{V}[\mathrm{kN}]$ is the shear resistance, $A\left[\mathrm{~mm}^{2}\right]$ is the adhesive surface of the laminated connections, $f_{V}[\mathrm{MPa}]$ represents the reference shear resistance that is equal to $5.53 \mathrm{MPa}$ for TSSA and $16.26 \mathrm{MPa}$ for SG, $\alpha_{V}[-]$ is the stress factor defined in the previous section, $\alpha_{\gamma}[-]$ is a factor that accounts for strain rate variation and $\alpha_{T}[-]$ is a factor that accounts for the temperature variations. In $\alpha_{\mathcal{E}}$ and $\alpha_{T}$, strain rate values are in [1/sec] and temperature values are in $\left[{ }^{\circ} \mathrm{C}\right]$.

$$
F_{V}=f_{V} \cdot \frac{1}{\alpha_{V}} \cdot \alpha_{\gamma} \cdot \alpha_{T} \cdot A
$$

Non-linear Matlab scripts are developed for the derivation of the model following a probabilistic approach. The scripts are developed to allow the derivation of (i) a model defined over one or more dimensions (in this case the two dimensions are strain rate and temperature) (ii) a model with a variable standard deviation and (iii) a model dependent on one or more parameters. The latter are described in details in the following two sections for TSSA and SG. The model it is assumed to follow a normal probability density function (pdf). However, different pdfs can be also implemented. The objective function of joint probability is then maximized within constrain functions.

Table 9 shows a first comparison between models and experimental results by means of the coefficient of determination, $R^{2}$, the mean and covariance of the model-test ratio. The following two sections compare more in detail the shear resistance given by the models to the experimental observations of TSSA and SG laminated connections under shear loading. Firstly, the model is directly compared with experiments with one-dimension plots. Secondly, the ratio between model and experiments is calculated and plotted for all investigated configurations of strain rate and temperature. These ratio plots in normalized scale allow to evaluate the accuracy of the model (deviation from value one) that might be not sufficiently clear in an absolute scale for all investigated test configurations. Thirdly, the residuals, i.e. difference between model and test results are calculated. This is done to analyse the variance of the model and namely to asses that the residuals have a random behaviour and tend to a normal distribution.

It should be noticed that the proposed models are to be used only within the investigated range of strain rate and temperature. Further analyses must be performed to extend the model out of the investigated ranges of strain rate and temperature.

Table 9: Summary of models-experimental comparisons

\begin{tabular}{|c|c|c|c|}
\hline \multirow{2}{*}{ Model } & \multicolumn{2}{|c|}{$F_{\text {model }} / F_{\text {test }}$} & \multirow{2}{*}{$\mathrm{R}^{2}$} \\
\hline & Mean & Cov & \\
\hline TSSA & 1.0050 & 0.0529 & 0.9065 \\
\hline SG & 1.0027 & 0.0496 & 0.9981 \\
\hline
\end{tabular}

\subsection{Model for TSSA laminated connections under shear load}

In this section the shear resistance given by the TSSA model are compared with the experimental results. The residual, calculated as difference between model and experimental observations, are also plotted. The strain rate factor proposed for the TSSA model is given by equation (14). The temperature factor proposed for the TSSA model is given by equation (15). More specifically, a logarithmic law is proposed for the effects of strain rate variation and linear law is instead proposed for the effects of temperature variation. 


$$
\begin{aligned}
& \alpha_{\gamma, T S S A}=1.27+\frac{\log (\dot{\gamma})}{10.59} \\
& \alpha_{T, T S S A}=1.04-\frac{T}{243.90}
\end{aligned}
$$

It is observed that the experimental results follow the proposed TSSA model for the full range of investigated temperatures (see Figure 24 (a) and Figure 25 (a)). One test result at $23^{\circ} \mathrm{C}$ seems to be outlier, with a normalized residual of approximately 13\% (see observation number 6 of Figure 24 (c) and the top of the plot of Figure 25(c)). The experimental results also follow the proposed TSSA model for the strain rate variation (see Figure 24 (b) Figure 25 (b)). A number of 6 specimens are tested at variable strain rates. More tests might be required to further confirm the strain rate effects. The plot of Figure 24 (c) and Figure 25(c) shows that the residuals have a random behaviour and tend to a normal distribution, which indicate a satisfactory representativeness of the model.

(a)

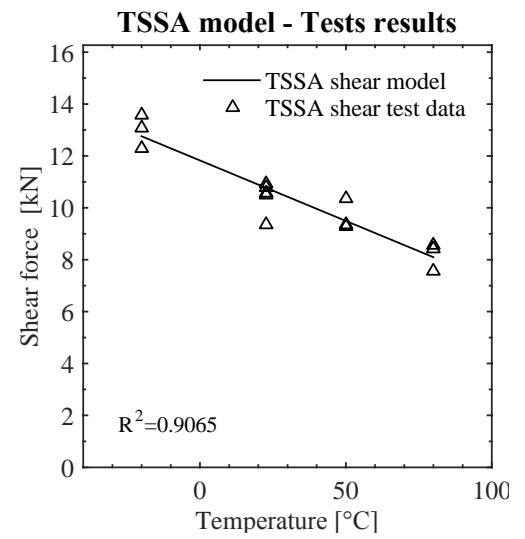

(b)

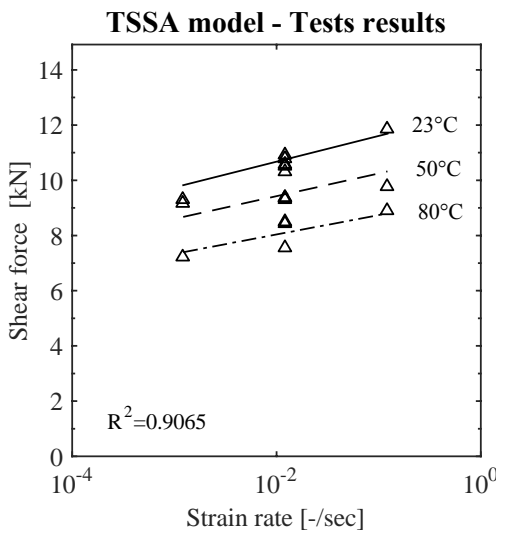

(c)

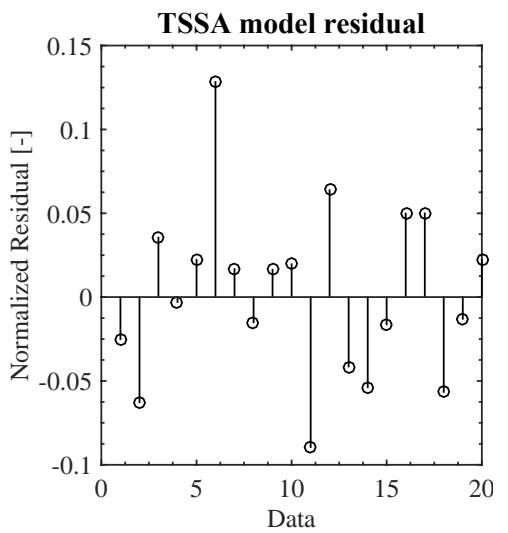

Figure 24: Comparison of the model with the experimental results versus (a) temperature for $1 \mathrm{~mm} / \mathrm{min}$ tests and (b) strain rate variations for $23^{\circ} \mathrm{C} 50$ and $80^{\circ} \mathrm{C}$ (continuous, dashed and dotted- dashed lines respectively)

(a)

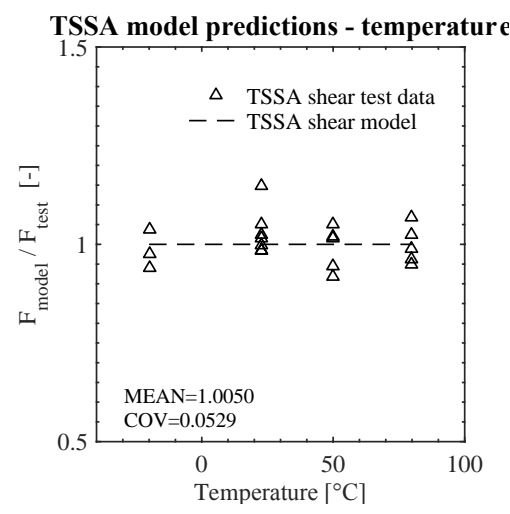

(b)

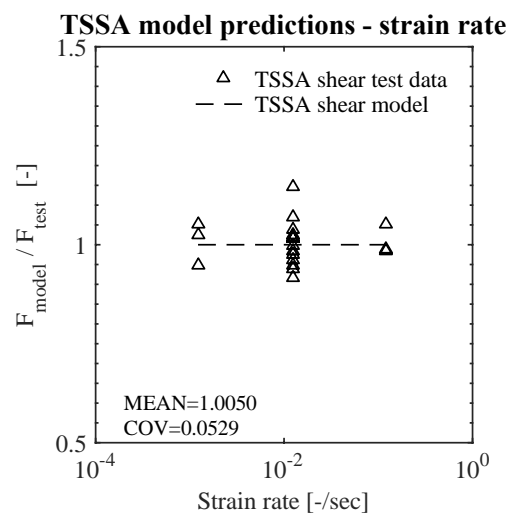

(c)

TSSA model residual

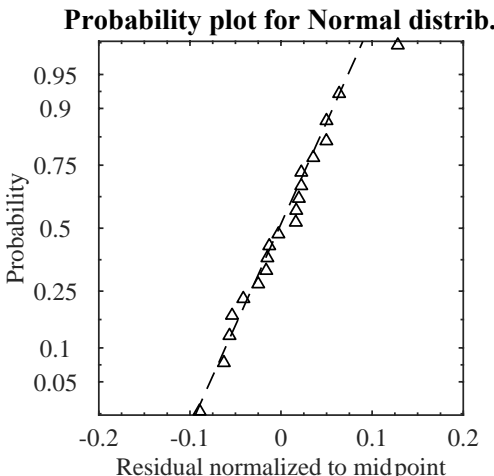

Figure 25: ratio of model-test for all the investigated configurations plotted versus (a) temperature (b) strain rate. (c) Probability plot of the residual for normal distribution.

\subsection{Model for SG laminated connections under shear load}

In this section the shear resistances given by the SG model are compared with the experimental results. The residuals, calculated as difference between model and experimental observations, are also plotted. The strain rate factor proposed for the SG model is given by equation (16). The temperature factor proposed for the SG model is given by equation (17). More specifically, a logarithmic law is proposed for the effects of strain rate variation. A mathematical expression based on inverse modified hyperbolic tangent function is instead proposed for the effects of temperature variation. 


$$
\begin{gathered}
\alpha_{\gamma, S G}=1.61+\frac{\log (\dot{\gamma})}{5.57} \\
\alpha_{T, S G}=0.32 \cdot\left(\tanh \left(\frac{28.17-T}{6.89}\right)+\tanh \left(\frac{25.27-T}{38.30}\right)+1.94\right)
\end{gathered}
$$

It is observed that the experimental results follow the proposed SG model for the full range of investigated temperatures, see Figure 26 (a) and Figure 27 (a). The experimental results also follow the proposed SG model for the strain rate variation, see Figure 26 (b) and Figure 27 (b). The model appears to be conservative in predicting the strain rate effect at high temperature. The resistance measured in the test is larger than the model (see outlier of Figure 26 (c) and Figure 27 (c)). In that regard, more tests might be required to further confirm the strain rate effect. A more complex expression of the strain rate factor might be developed which accounts for quadratic logarithmic term and for temperature variation inside the strain rate factor itself. The plot of Figure 26 (c) and Figure 27 (c) shows that the residuals have a random behaviour and tend to a normal distribution.

(a)

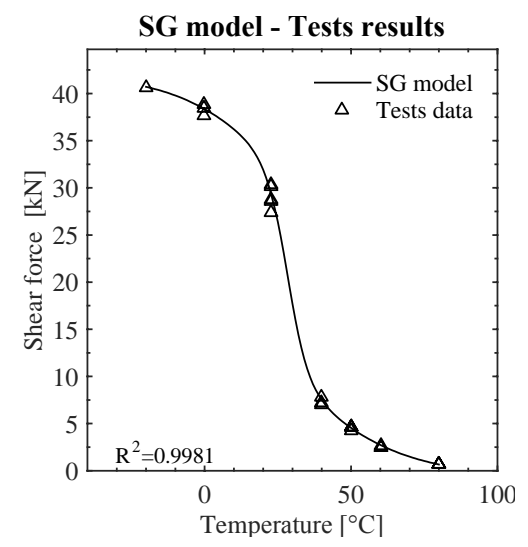

(b)

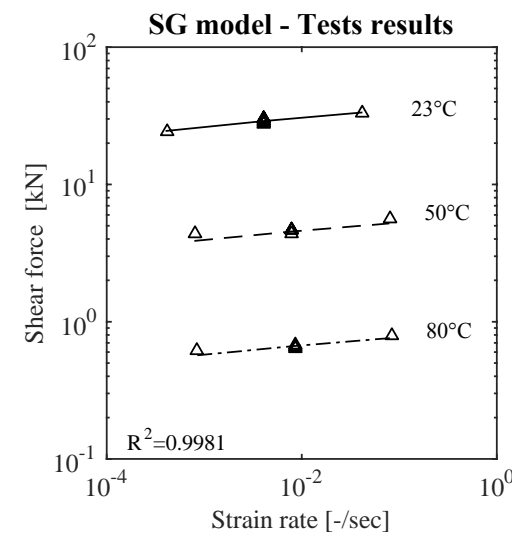

(c)

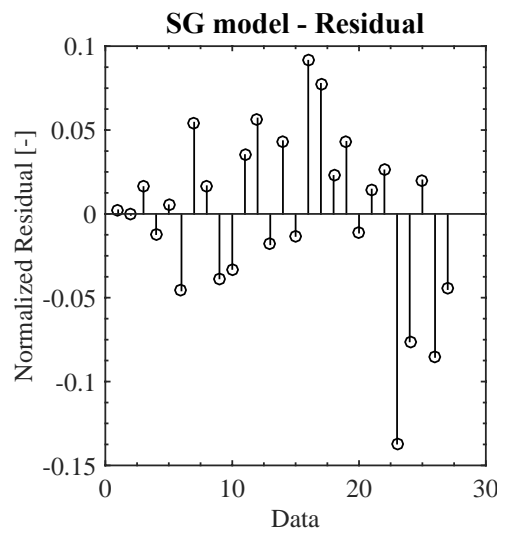

Figure 26: Comparison of the model with the experimental results versus (a) temperature for $1 \mathrm{~mm} / \mathrm{min}$ tests and (b) strain rate variations for $23^{\circ} \mathrm{C} 50$ and $80^{\circ} \mathrm{C}$ (continuous, dashed and dotted- dashed lines respectively)

(a)

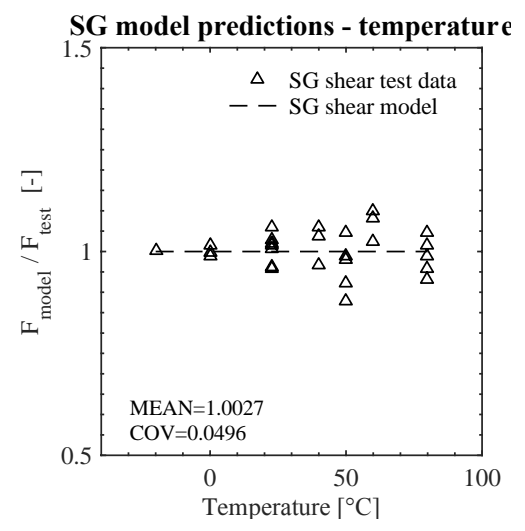

(b)

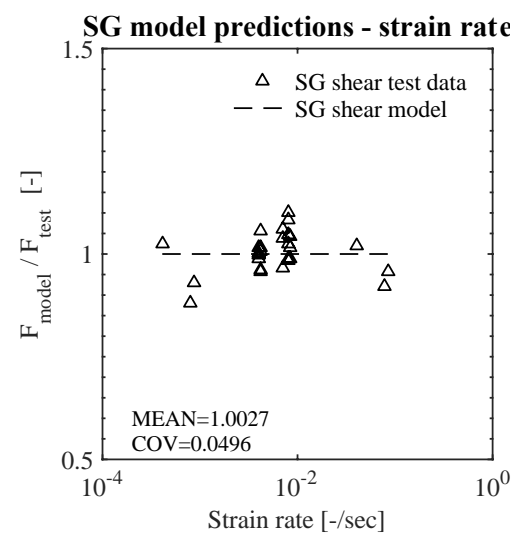

(c)

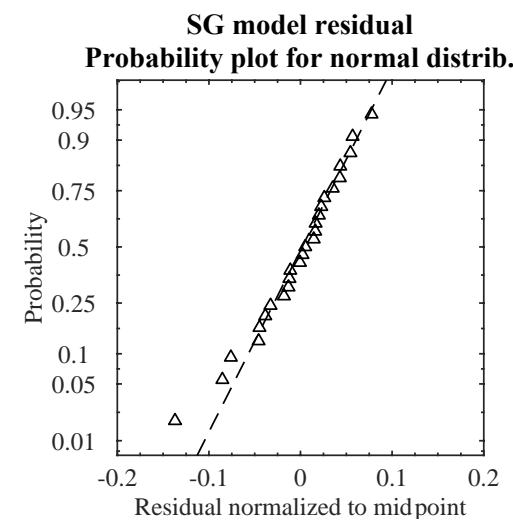

Figure 27: ratio of model-test for all the investigated configurations plotted versus (a) temperature (b) strain rate. (c) Probability plot of the residual for normal distribution. 


\section{Conclusions}

The experimental investigations presented in this work showed that temperature and strain rate variations have important effects on the mechanical response of laminated connection under shear load. The resistance is proportional to the logarithm of the strain rate, decreases at high temperature and increases at low temperature for both materials. The failure mode is always cohesive for both materials. In laminated SG connections tested at room temperature and low temperature, glass failure is also observed. More specifically, contrarily to the expectations, stable glass crack in tempered panel is sometimes observed. The stable crack initiates approximately at the middle of the connection and propagates through thickness with a 'drop-like' shape. It is observed that the fracture propagates in a typical explosive manner only in the cases where the crack depth exceeds $20 \%$ of the thickness. The used of favourable residual stress should be considered with caution, since three-dimensional anisotropy might play an important role when the direction of the principal stress is not parallel to the glass plate.

Three-dimensional numerical analyses are performed to determine the stress distribution in the connection. Firstly, it is observed that the stress field shows large gradient over the three dimensions. It is stated that accurate three-dimensional models, which account for three-dimensional effects, complex geometry and accurate boundary conditions, must be used. Secondly, it is stated that the stress distribution in the adhesive is non-linear and exhibits stress peaks. These stress peaks are located close to the free edges and that theirs magnitudes depend on the adhesive stiffness, which is a function of the temperature. A parametrical study is so performed to quantify the temperature effect on the stress peaks. This is done by the definition of a so-called stress factor that describes the deviation of actual stress from the nominal one. Thirdly, the numerical results are compared to the glass crack pattern observed in the experimental investigation. It is shown that the location and the 'drop-like' shape of the stable glass crack confirm the three-dimensional stress distributions computed by the finite element analyses.

A simplified two-dimensional variational approach is used to analytically explain the non-linearity of the stress field in the adhesive. It is derived that ratio between adherends and adhesive plays an important role in the occurring of stress peaks. In addition to that, it is also analytically observed that, after a certain extent, the increase of overlapping length (radius of the metal connector in this problem) is not an efficient measure to increase the joint resistance with very stiff adhesives. This effect is less pronounced with medium and soft adhesives.

Finally, prediction models are proposed for the shear resistance of TSSA and SG laminated connections. The models are obtained developing a Matlab algorithm that allows the derivation of multi-dimensional non-linear models with variable standard deviations. It is observed that the models are able to predict the behaviour of the laminated connections for the full range of investigated strain rates and temperatures. More specifically, a logaritmic law is proposed for the strain rate effects for both TSSA and SG connections. Linear and inverse hyperbolic-tangent-based laws are instead proposed for the TSSA and SG temperature behaviour respectively.

\section{Acknowledgements}

The authors would like to thank the Swiss National Science Foundation for founding the present research (Grants 200020_150152 and 200021_134507). In addition, the COST Action TU0905 "Structural Glass - Novel Design Methods and Next Generation Products" is also acknowledged for facilitating the research network. 


\section{References}

[1] L. Blandini, "Structural Use of Adhesive in Glass Shells," Thesis Dissertation, University of Stuttgart, 2005.

[2] J. Belis, A. Van Hulle, D. Callewaert, and J. Dispersyn, "Experimental investigation of unconventional canopy prototypes, suspended by adhesive bonds," in Challenging Glass 3, 2012.

[3] V. Dias, O. Hechler, and C. Odenbreit, "Determination of adhesives properties for non-linear numerical simulation of structural steel-glass connections," in Challenging Glass 3, 2012.

[4] J. Watson, "Premature Failure in UV-Cured Adhesive Joints," in ISAAG, 2010.

[5] E. M. P. Huveners, F. Van Herwijnen, F. Soetens, and H. Hofmeyer, "Mechanical shear properties of adhesives," in Glass Performance Days, 2007.

[6] V. A. Silvestru and O. Englhardt, "Application study for hybrid adhesively bonded glass-steel façade elements," in Challenging Glass 4 \& COST Action TU0905 Final Conference, 2014.

[7] S. Marinitsch and C. Schranz, "Folded plate structures made of glass laminates : a proposal for the structural assessment," Glas. Struct. Eng., 2015.

[8] J. Dispersyn, J. Belis, and D. Sonck, "New glass design method for adhesive point-fixing applications," Proc. ICE - Struct. Build., 2015.

[9] E. Speranzini, S. Agnetti, and M. Corradi, "Experimental analysis of adhesion phenomena in fibre-reinforced glass structures," Compos. Part B Eng., vol. 101, pp. 155-166, 2016.

[10] B. Weller and I. Vogt, "Adhesive Joints in Glass and Solar Engineering," J. ASTM Int., vol. 9, no. 5, p. 104076, May 2012.

[11] B. Weller, F. Nicklisch, V. Prautzsch, and I. Vogt, "Outline of Testing and Evaluation Program Used in Selection of Adhesives for Transparent Adhesive Joints in All-Glass Load-Bearing Structures," J. ASTM Int., vol. 9, no. 4, p. 104088, Apr. 2012.

[12] M. Santarsiero, C. Louter, and A. Nussbaumer, "The mechanical behaviour of SentryGlas ionomer and TSSA silicon bulk materials at different temperatures and strain rates under uniaxial tensile stress state," Glas. Struct. Eng., 2016.

[13] J. O'Callaghan and G. Coult, "An all glass cube in New York City," in Glass Performance Days, 2007.

[14] J. O'Callaghan, “Thinking big with structural glass,” in Glass Performance Days, 2009.

[15] J. O'Callaghan, “Adventures with Structural Glass,” in Glass Performance Days, 2012.

[16] P. Lenk and H. Lambert, "Challenges in the design, fabrication and installation of glass structures comprising of super jumbo glass sheets," in Challenging Glass 3, 2012.

[17] P. Lenk and F. Lancaster, "Connections in structural glass," in Glass Performance Days, 2013.

[18] J. Ludwig, "Tottenham Court Road Station - Plaza entrances structures and building structural glass," in Challenging Glass 4 \& COST Action TU0905 Final Conference, 2014.

[19] S. Sitte and A. Wolf, "TSSA-bonded point-supported structural glazing of insulating glass units at Dow Corning's European Distribution Center," in Engineered Transparency, 2012.

[20] Kuraray and obtained through web site http://glasslaminatingsolutions.kuraray.com, "SentryGlas ${ }^{\circledR}$ - data sheet." 2009.

[21] I. Stelzer, "High Performance Laminated Glass," in Challenging Glass 2, 2010.

[22] Dow Corning and obtained through website www.dowcorning.com, "TSSA - Transparent Structural Silicone Adhesive - data sheet." 2013.

[23] CEN - European Committee for Standardisation, "EN 572 - 1 - Glass in building - Basic soda 
lime silicate glass products - Part 1: Definitions and general physical and mechanical properties." 2004.

[24] CEN - European Committee for Standardisation, "EN 572 - 2 - Glass in building - Basic soda lime silicate glass products - Part 2: Float glass.”.

[25] M. Haldimann, A. Luible, and M. Overend, Structural Use of Glass. International Association for Bridge and Structural Engineering - IABSE, 2008.

[26] CEN - European Committee for Standardisation, "Eurocode 3: Design of steel structures - Part 1-4: General rules - Supplementary rules for stainless steels.".

[27] CEN - European Committee for Standardisation, "EN 10088-2 - Stainless steel - Part 2: Technical delivery conditions for sheet/plate and strip of corrosion resisting steels for general purposes.”.

[28] R. Decourcelle, J. C. Nugue, and F. Levasseur, "Mechanical participation of interlayer on laminated glass for building applications," in Glass Performance Days, 2009, pp. 718-721.

[29] L. Calgeer, "Structural design of glass façade components: comparison of different connection typologies," Master Thesis Dissertation, École Polytechnique Fédérale De Lausanne - EPFL, 2015.

[30] R. Varley, "Ionomers as Self Healing Polymers," in Self Healing Materials. An Alternative Approach to 20 Centuries of Materials Science, S. van der Zwaag, Ed. Springer, 2007, pp. 95114.

[31] A. Eisenberg and M. Rinaudo, "Polyelectrolytes and ionomers," Polym. Bull., vol. 671, 1990.

[32] W. J. Macknight and J. Earnest, "The structure of ionomer," J. Polym. Sci. Macromol. Rev., vol. 19, pp. 41-122, 1981.

[33] R. Decourcelle and F. Levasseur, "Long term behaviour of laminated glass plates by bending creep experiments," in ISAAG, 2010.

[34] D. Delincé, "Experimental Approaches for Assessing Time and Temperature Dependent Performances of Fractured Laminated Safety Glass," Thesis Dissertation, Ghent University, 2014.

[35] S. J. Bennison and P. S. Davies, "High-performance laminated glass for structurally efficient glazing," in Innovative light-weight structures and sustainable façades, 2008.

[36] J. Belis, J. Vander Beken, R. Van Impe, and D. Callewaert, "Performance of glass-ionoplast laminates above room temperature," in Glass Performance Days, 2007.

[37] D. Callewaert, D. Delincé, J. Belis, and R. Van Impe, "Temperature-dependent behaviour of glass / ionomer laminates : preliminary test results," in Challenging Glass, 2008.

[38] D. Callewaert, "Stiffness of glass/ionomer laminates in structural application," Thesis Dissertation, Ghent University, 2011.

[39] D. Callewaert, J. Belis, D. Delincé, and R. Van Impe, "Experimental stiffness characterisation of glass/ionomer laminates for structural applications," Constr. Build. Mater., vol. 37, pp. 685692, 2012.

[40] J. D. Ferry, Viscoelastic properties of polymers. Wiley \& Sons.

[41] M. L. Williams, R. F. Landel, and J. D. Ferry, "The temperature dependence of relaxation mechanisms in amorphous polymers and other glass-forming liquids," J. Am. Chem. Soc., vol. 77, no. 14, pp. 3701-3707, 1955.

[42] J. Belis, "Kipsterkte van monolithische en gelamineerde glazen liggers," Thesis Dissertation, Ghent University, 2005.

[43] C. Bedon, J. Belis, and A. Luible, "Assessment of existing analytical models for the lateral torsional buckling analysis of PVB and SG laminated glass beams via viscoelastic simulations and experiments," Eng. Struct., vol. 60, pp. 52-67, 2014. 
[44] M. Santarsiero, C. Louter, and A. Nussbaumer, "Laminated connections for structural glass components : a full-scale experimental study," Glas. Struct. Eng., 2016.

[45] J. Schneider, S. Kolling, J. Kuntsche, and S. Moennich, "Tensile properties of different polymer interlayers under high strain rates," in Engineered Transparency, 2012.

[46] K. Puller, J. Denonville, and W. Sobek, "An innovative glass connection technique using an ionomer interlayer," in Glass Performance Days, 2011.

[47] M. Meissner and V. Sackmann, "On the Effect of Artificial Weathering on the Shear Bond and the Tear Strength of two different Interlayers of Laminated Glass," in ISAAG, 2006.

[48] J. Kuntsche and J. Schneider, "Mechanical behaviour of polymer interlayers in explosion resistant glazing," in Challenging Glass 4 \& COST Action TU0905 Final Conference, 2014, pp. 447-454.

[49] L. Biolzi, S. Cattaneo, and G. Rosati, "Progressive damage and fracture of laminated glass beams," Constr. Build. Mater., vol. 24, no. 4, pp. 577-584, 2010.

[50] S. J. Bennison, J. G. Sloan, D. F. Kristunas, P. J. Buehler, T. Amos, and C. A. Smith, "Laminated Glass for Blast Mitigation: Role of Interlayer Properties," in Glass Processing Days, 2005.

[51] J. Belis, J. Depauw, D. Callewaert, D. Delincé, and R. Van Impe, "Failure mechanisms and residual capacity of annealed glass/SGP laminated beams at room temperature," Eng. Fail. Anal., vol. 16, no. 6, pp. 1866-1875, 2009.

[52] S. Peters, A. Fuchs, J. Knippers, and S. Behling, "Ganzglastreppe mit transparenten SGPKlebeverbindungen - Konstruktion und statische Berechnung," Stahlbau, vol. 76, no. 3, pp. 151-156, Mar. 2007.

[53] J. Belis, A. Van Hulle, B. Out, F. Bos, D. Callewaert, and H. Poulis, "Broad screening of adhesives for glass-metal bonds," in Glass Performance Days, 2011, pp. 286-289.

[54] A. Van Hulle, J. Belis, D. Callewaert, L. Scheerlinck, and B. Out, "Development of structural adhesive point-fixings," in Glass Performance Days, 2011.

[55] J. Belis, D. Callewaert, and A. Van Hulle, Bouwen met glas en adhesieven - praktische gids voor ontwerper en uitvoerder. Universiteit Gent, Laboratorium voor modelonderzoek, 2012.

[56] J. Watson, "Novel Structural Glass Connections for Architectural Applications," Thesis Dissertation, University of Cambridge, 2013.

[57] K. Puller, "Untersuchung des Tragverhaltens von in die Zwischenschicht von Verbundglas integrierten Lasteinleitungselementen," Thesis Dissertation, University of Stuttgart, 2012.

[58] K. Puller and W. Sobek, "Load-carrying behaviour of metal inserts embedded in laminated glass," in Challenging Glass 3, 2012.

[59] P. Carvalho, P. J. S. Cruz, and F. Veer, "Perforated steel plate to laminated glass adhesive properties," in Glass Performance Days, 2011.

[60] P. Carvalho and P. J. S. Cruz, "Connecting Through Reinforcement - Experimental Analysis of A Glass Connection Using Perforated Steel Plates," in Challenging Glass 3, 2012.

[61] M. Santarsiero, P. Carvalho, C. Louter, and P. J. S. Cruz, "Experimental and numerical investigations of metal-to-glass embedded connections with thin stainless steel plate," in COST Action TU0905, Mid-term Conference on Structural Glass, 2013.

[62] J. Ludwig, "Tottenham Court Road Station Upgrade - Structural Glass Plazas," in International Conference on Building Envelope Design and Technology, 2015.

[63] M. Santarsiero and C. Louter, "Embedded and point laminated adhesive connections for glass structures : parametric non-linear numerical investigations," in Glass Performance Days, 2013, pp. $265-273$.

[64] S. Sitte, M. J. Brasseur, L. D. Carbary, and A. T. Wolf, "Preliminary Evaluation of the Mechanical Properties and Durability of Transparent Structural Silicone Adhesive ( TSSA ) for 
Point Fixing in Glazing,” J. ASTM Int., vol. 8, no. 10, pp. 1-27, 2011.

[65] S. Sitte, "Experimental evaluation of mechanical properties of TSSA," 2011.

[66] A. T. Wolf and K. Hirai, "Evaluation of transparent structural silicone film adhesive for bonded point-fixed glazing," in Engineered Transparency, 2010, pp. 167-177.

[67] P. Vandereecken, M. Elliott, and M. Plettau, "Durable high design strength in glass curtain wall," in Challenging Glass 4 \& COST Action TU0905 Final Conference, 2014, pp. 401-408.

[68] European Organisation for Technical Approvals, "ETAG 002 - Guideline for European Technical approval for Structural Sealant Glazing Systems ( SSGS ) Part 1: Supported and unsupported system." 2001.

[69] A. Hagl, O. Dieterich, A. T. Wolf, and S. Sitte, "Tensile Loading of Silicone Point Supports Revisited," in Challenging Glass 3, 2012.

[70] A. Hagl, A. T. Wolf, and S. Sitte, "Investigation of Stress-Whitening in Transparent Structural Silicon Adhesive," in Challenging Glass 3, 2012.

[71] M. Santarsiero, "Laminated connections for structural glass applications," Thesis Dissertation, École Polytechnique Fédérale De Lausanne - EPFL, 2015.

[72] R. D. Adams, J. Comyn, and W. C. Wake, Structural adhesive joints in engineering. Chapman \& Hall, 1997.

[73] D. a. Bigwood and a. D. Crocombe, "Elastic analysis and engineering design formulae for bonded joints," Int. J. Adhes. Adhes., vol. 9, no. 4, pp. 229-242, 1989.

[74] SIMULIA, Abaqus Analysis User's Manual - Volume IV: Elements, vol. IV. 2011. 


\section{SG shear test results}
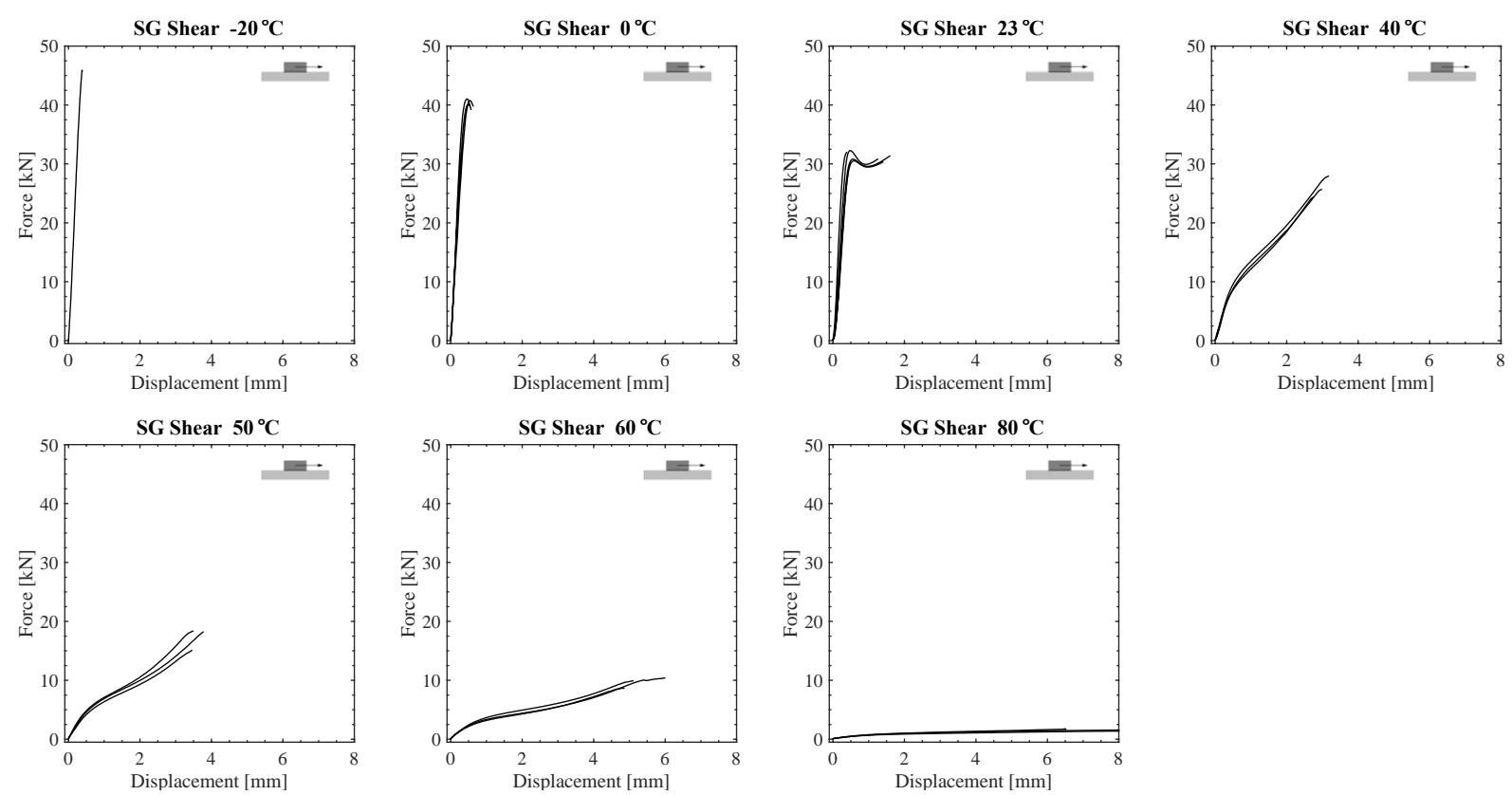

\section{TSSA shear test results}
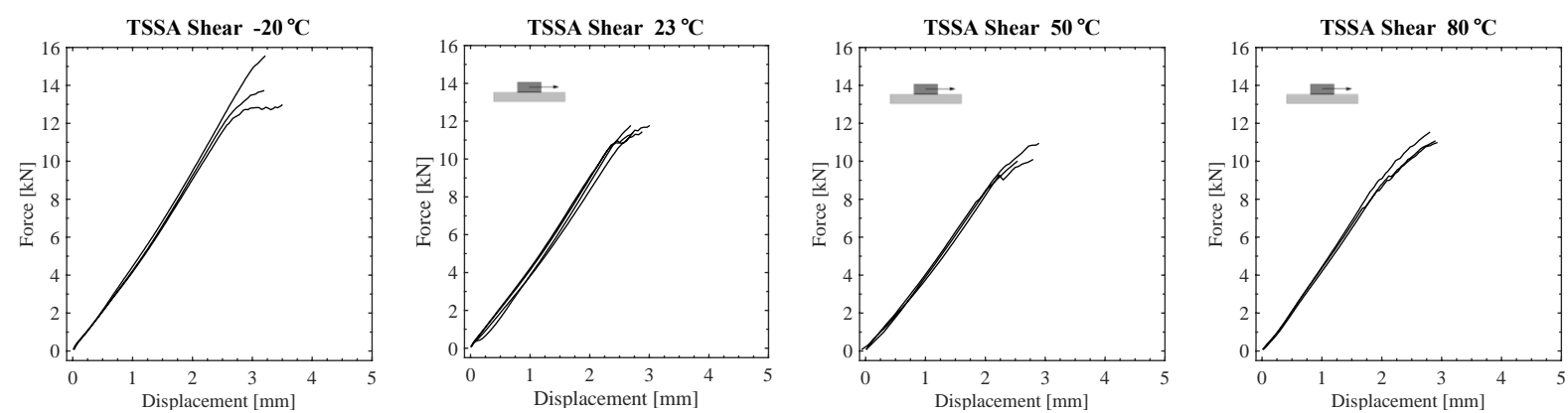


\section{Appendix B - Solver, element and mesh study}

This Appendix describes the analyses performed for the choice of the solver, element and mesh size. A total number of 11 different cases are analysed. Table 10 collects the detail for each case. Cases from 1 to 5 regard mesh size, case 6 the solver algorithm, cases from 7 to 11 the element type, element order and element formulation. The comparison parameter used for the mesh study is the size of the "local mesh seeds constrain" given to the meshing algorithm for the adhesive region. The results of each analysis are summarized by Table 11 and then discussed. The case adopted in the analyses of this work is case number 8 . The geometry of the model is the same use in this work. Figure 28 shows the mesh pattern used to perform the mesh and element study. Other parameters kept constant for all analyses are listed below:

- Applied force $=2 \mathrm{kN}$

- Relative tolerance of convergence (iterative solver)=1e-6

- Residual force convergence criterion=1e-6

- Displacement convergence criterion=1e-6

- $E=600 \mathrm{MPa}, v=0.43$

(a)

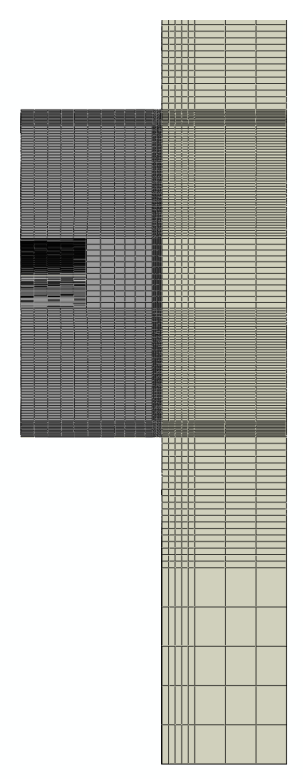

(b)

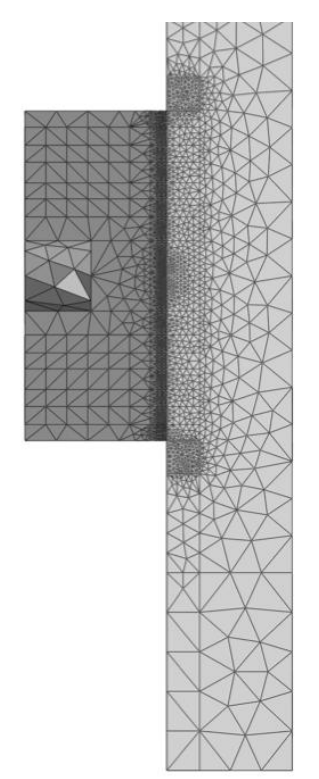

Figure 28: Mesh patterns use in the solver, element and mesh study: (a) using hexahedron elements (b) using tetrahedral elements.

Same model geometry and approximately same element size constrains lead to an average aspect ratio of 3-6 for hexahedron and 1.65 for tetrahedral meshing algorithms. The aspect ratio of hexahedron is excessive since the performances of this element are very poor when they are elongated.

It is observed that hexahedron "sweep" meshing algorithm often does not converge for this problem, especially with small mesh size. Instead, hexahedron "structured" algorithm usually converge. However, it does not permit a gradual mesh size refinement going toward the adhesive where fine meshing is required. The mesh size at the adhesive is instead propagated also to glass and metal (see Figure 28 (a)). This leads to poor element aspect ratio. Tetrahedral meshing algorithm always converges for this problem. In principle, more tetrahedral elements are needed to cover the same volume than with hexahedron. However, tetrahedral permits a gradual mesh size refinement going from glass and steel towards the adhesive. This results in reduced number of elements. This also leads 
to better element aspect ratio. Given the same problem and same mesh size constrain, approximately $700 \mathrm{k}$ and $400 \mathrm{k}$ elements are needed to model the connection respectively with hexahedron and tetrahedral meshing algorithm. Because of the reduced degrees of freedom, reduced CPU time and better aspect ratio, tetrahedral are preferred to hexahedron for this problem. Second order elements are usually preferred. In the case convergency is not achievable, (e.g. very large deformation) first-order elements and the mesh is further reduced [74]. A control of the pressure profile and displacement field is performed to ensure that volumetric locking is not occurring.

(a)

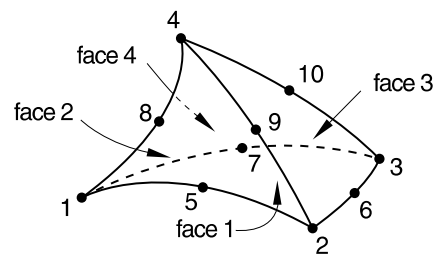

(b)

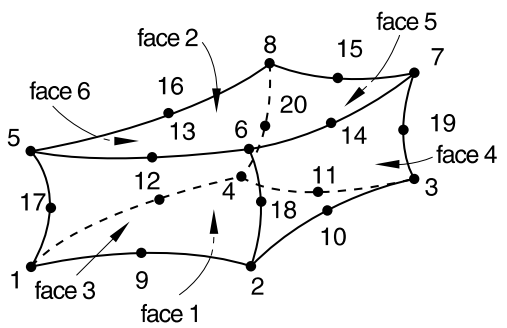

Figure 29: (a) tetrahedral element (b) hexahedron element used in the solver, element and mesh study [74]

The stiffness matrix of three-dimensional solid model is generally not concentrated along the diagonal. For such type of problem the direct sparse solver algorithm might be not competitive against iterative solver. See Table 11, case 5 and 6 . Iterative solver algorithm is therefore adopted for the current problem.

Modelling soft material connected to stiff material is by nature numerically ill-conditioned problem. In this case, large numbers of iterations are needed to converge to the solution. In addition to that, the mesh refinement here required to capture the adhesive stress intensification leads to large difference in the elements sizes. The element stiffness is therefore different even in case of similar material. This is a second source of numerical ill-conditioning that increases the number of iteration needed to achieve convergence. For the current problem, the available calculation power is enough to keep the solving time reasonable. In case of larger problem, the sub-modelling approach might be considered as an efficient alternative. Additionally to these ill-conditioning sources, the computation cost is also increased (i) because very large deformations are in some cases involved (e.g. TSSA or SG at high temperature) and (ii) because finite deformation theory is used to account for geometrical nonlinearity. Because of that, convergence is often difficult to achieve and large number of iterations are usually required depending on the problem. The required number of iteration is usually large and therefore double precision is here adopted to reduce numerical truncation errors. 
Table 10: Solver, element and mesh study: cases descriptions

\begin{tabular}{ccccc}
\hline Case & Element type & Solver algorithm & Mesh seeds & Elements order \\
\hline 1 & Tetrahedral & iterative & $5.5^{*}$ & first-order \\
2 & Tetrahedral & iterative & 1 & first-order \\
3 & Tetrahedral & iterative & 0.5 & first-order \\
4 & Tetrahedral & iterative & 0.1 & first-order \\
5 & Tetrahedral & iterative & 0.2 & first-order \\
6 & Tetrahedral & direct & 0.2 & first-order \\
7 & Hexahedron reduced integration & iterative & 0.2 & first-order \\
8 & Tetrahedral & iterative & 0.2 & second-order \\
9 & Tetrahedral modified & iterative & 0.2 & second-order \\
10 & Tetrahedral improved stress surface & iterative & 0.2 & second-order \\
11 & Tetrahedral hybrid & iterative & 0.2 & first-order \\
\hline
\end{tabular}

For cases from 5 to 11 the mesh is locally refined close to the adhesive also for the glass and the metal connector *only global mesh seed constrain is given.

Table 11: Solver, element and mesh study: cases results

\begin{tabular}{cccccccc}
\hline Case & Variation [\%] & \# Element & D.o.f. & CPU time & Total time & $\begin{array}{c}\text { Stress field } \\
\text { continuity }\end{array}$ & $\begin{array}{c}\text { Elements } \\
\text { aspect ratio }\end{array}$ \\
\hline 1 & 8.60 & 13454 & 9201 & 4.8 & $00: 17$ & very poor & poor \\
2 & 8.94 & 38408 & 22941 & 12 & $00: 25$ & very poor & poor \\
3 & 6.29 & 170048 & 91638 & 62 & $01: 30$ & poor & ok \\
4 & 5.43 & 388241 & 217677 & 170 & $04: 00$ & ok & ok \\
5 & 3.75 & 718355 & 380844 & 292 & $06: 40$ & ok & ok \\
6 & 3.75 & 718355 & 380844 & 1395 & $09: 00$ & ok & ok \\
7 & 1.69 & 242808 & 790281 & 1315 & $23: 00$ & ok & very poor \\
8 & 0.24 & 718355 & 2960478 & 6497 & $02: 04: 00$ & ok & ok \\
9 & 0.00 & 718355 & 5115543 & 5864 & $02: 10: 00$ & ok & ok \\
10 & $*$ & 718355 & 3085022 & $*$ & $*$ & * & ok \\
11 & $*$ & 718355 & 505388 & $*$ & $*$ & * & ok \\
\hline
\end{tabular}

*not converged 
Solver, elment and mesh study - Deegrees of freedom

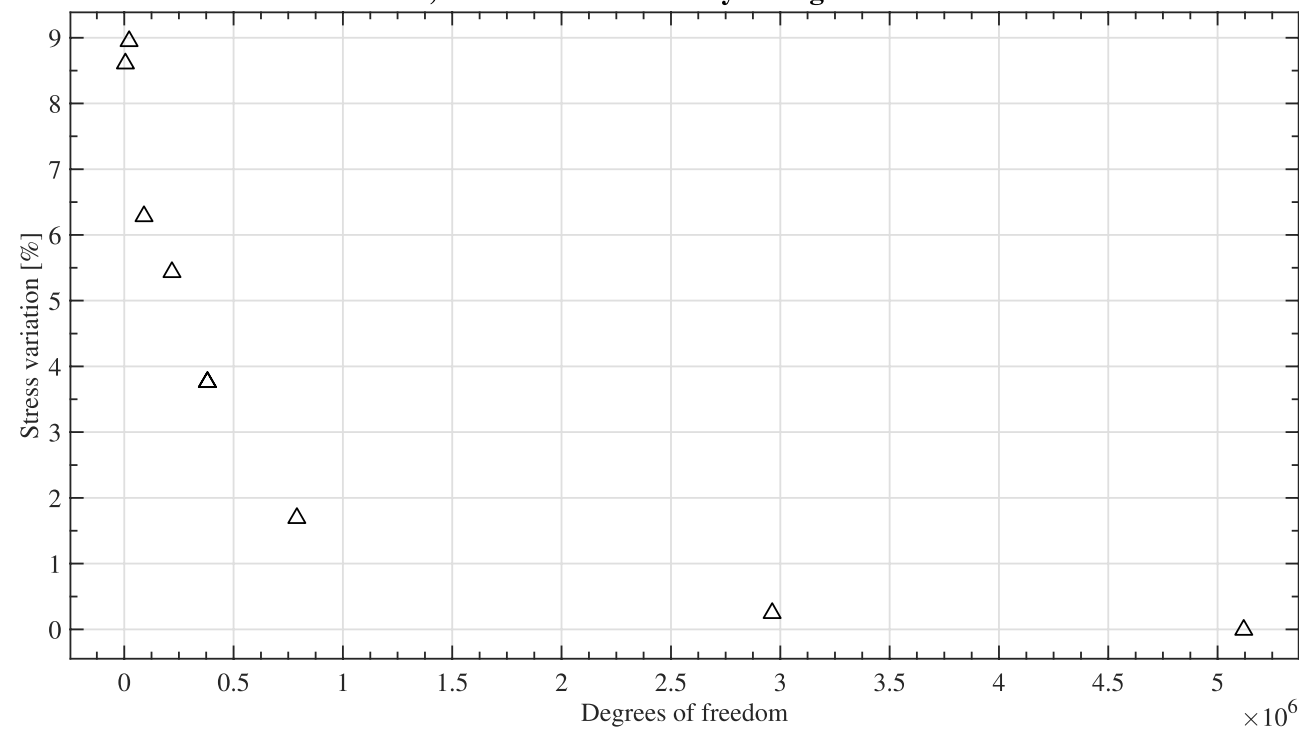

Figure 30: Stress variation versus number of degrees of freedom of the model (millions)

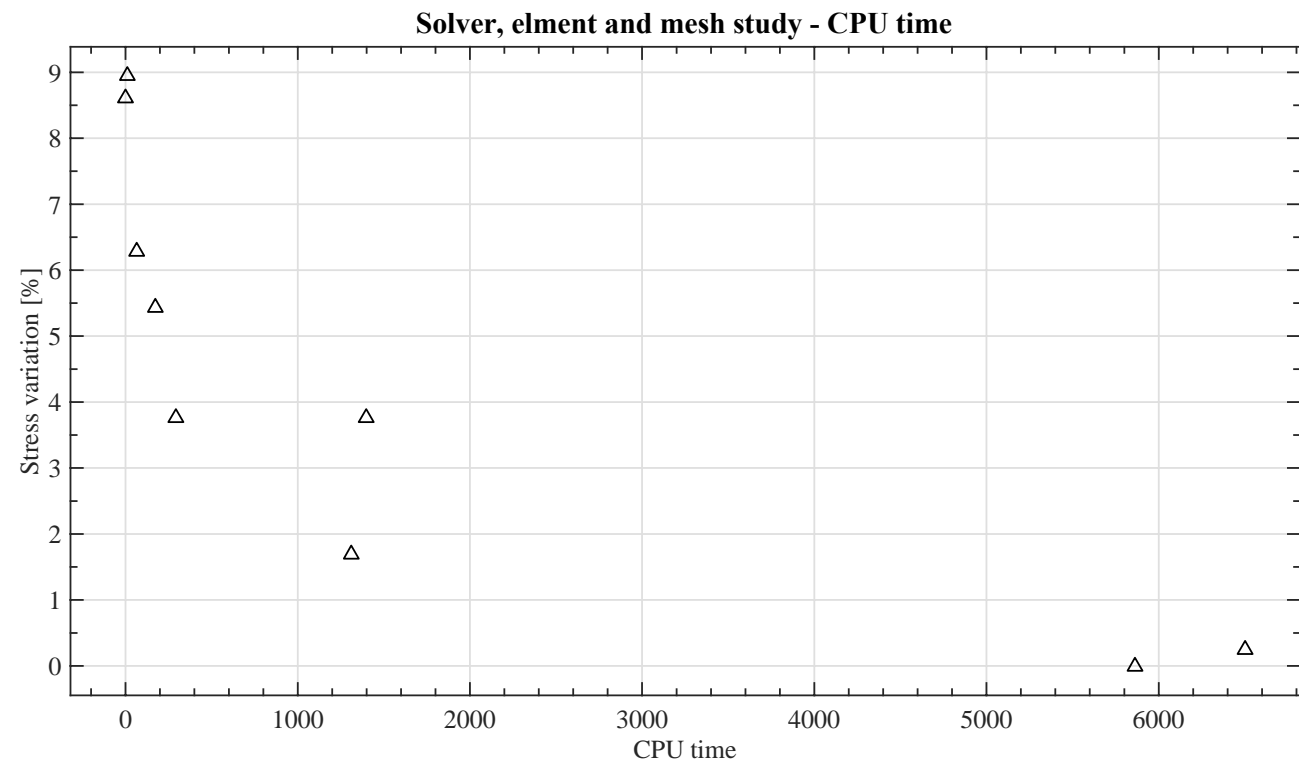

Figure 31: Stress variation versus needed CPU time (sec) to achieve to converged solution 


\section{Appendix C - Residual stress profile of $19 \mathrm{~mm}$ fully tempered glass}

In this appendix, the residual stress profile of a $19 \mathrm{~mm}$ fully tempered glass is presented. The dimensions of the glass plate are $300 \times 150 \mathrm{~mm}$. The measurements are performed using a SCALP 05 up to $5 \mathrm{~mm}$ form the surface. The computation algorithm assumes ${ }^{18}$ hypotheses of uniformity, isotropy and plane stress condition of the residual stress field distribution. The measurements are performed at $75 \mathrm{~mm}$ distance from both edges. Measurements are repeated 10 times at the same location. The residual stress profile is plotted in Figure 32. The surface residual stress is given in Table 12. Tensile zone is measured to be at $3.54 \mathrm{~mm}$ from the surface, which corresponds to $19 \%$ of the glass nominal thickness.

Table 12: Measurements of the surface residual stress of a 19mm fully tempered glass panel of a SG laminated connections

\begin{tabular}{ccc}
\hline Mean & -105.08 & $\mathrm{MPa}$ \\
St.dev & 0.31 & $\mathrm{MPa}$ \\
COV & 0.3 & $\%$ \\
\hline
\end{tabular}

(a)

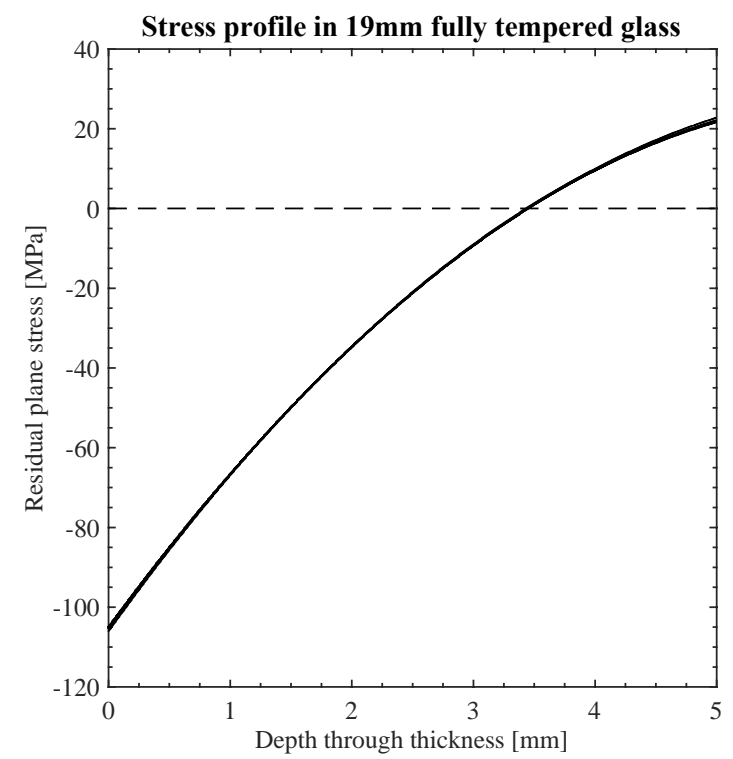

(b)

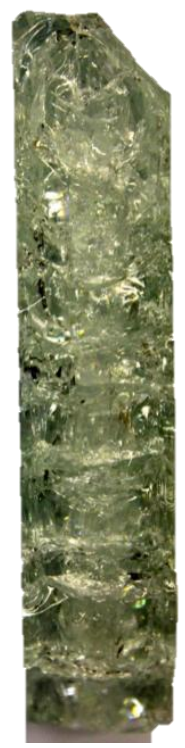

(c)

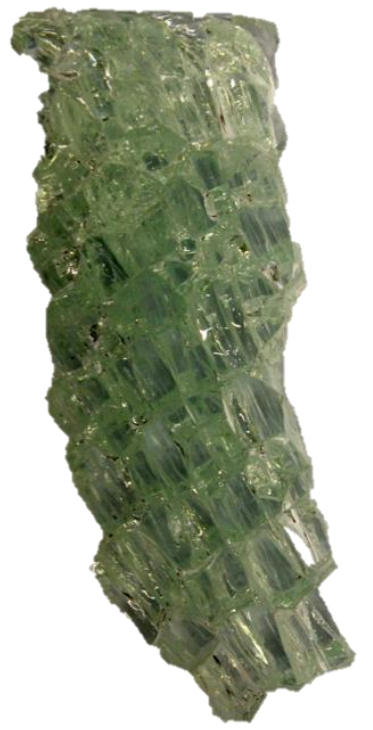

Figure 32: (a) Profile of residual of a 19mm fully tempered glass panel (b) through thickness view of a broken $19 \mathrm{~mm}$ fully tempered glass panel of a SG laminated connections after shear test: lighter internal region indicate the tensile region of the residual stress profile (c) Plan view of a broken $19 \mathrm{~mm}$ fully tempered glass panel of a SG laminated connections after shear test.

\footnotetext{
${ }^{18}$ Isotropy and uniformity hypothesis has been experimentally verified in [29] being not exceeding 5\% variation.
} 
